\title{
Modeling semantic aspects for cross-media image indexing
}

Florent Monay and Daniel Gatica-Perez

$\{$ monay, gatica $\} @$ idiap.ch

IDIAP Research Institute and Ecole Polytechnique Federale de Lausanne Rue du Simplon 4, Case Postale 592, 1920 Martigny, Switzerland 


\begin{abstract}
To go beyond the query-by-example paradigm in image retrieval, there is a need for semantic indexing of large image collections for intuitive text-based image search. Different models have been proposed to learn the dependencies between the visual content of an image set and the associated text captions, then allowing for the automatic creation of semantic indices for unannotated images. The task, however, remains unsolved. In this paper, we present three alternatives to learn a Probabilistic Latent Semantic Analysis model (PLSA) for annotated images, and evaluate their respective performance for automatic image indexing. Under the PLSA assumptions, an image is modeled as a mixture of latent aspects that generates both image features and text captions, and we investigate three ways to learn the mixture of aspects. We also propose a more discriminative image representation than the traditional Blob histogram, concatenating quantized local color information and quantized local texture descriptors. The first learning procedure of a PLSA model for annotated images is a standard EM algorithm, which implicitly assumes that the visual and the textual modalities can be treated equivalently. The other two models are based on an asymmetric PLSA learning, allowing to constrain the definition of the latent space on the visual or on the textual modality. We demonstrate that the textual modality is more appropriate to learn a semantically meaningful latent space, which translates into improved annotation performance. A comparison of our learning algorithms with respect to recent methods on a standard dataset is presented, and a detailed evaluation of the performance shows the validity of our framework.
\end{abstract}

\title{
Index Terms
}

Image annotation, textual indexing, image retrieval, quantized local descriptors, latent aspect modeling.

\section{INTRODUCTION}

With the production of large digital image collections favored by cheap digital recording and storage devices, there is a clear need for efficient indexing and retrieval systems. The ideal system should allow for intuitive search for the user, and require a minimal amount of human interaction to be applicable to large collections. Two distinct approaches to search large image collections coexist in the literature.

One is based on the query-by-example (QBE) paradigm [24], [32], [33], [7], [30]. In QBE systems, various low-level visual features are preliminarily extracted from the dataset and stored as image indices. The query is an image example that is indexed by its features, and retrieved 


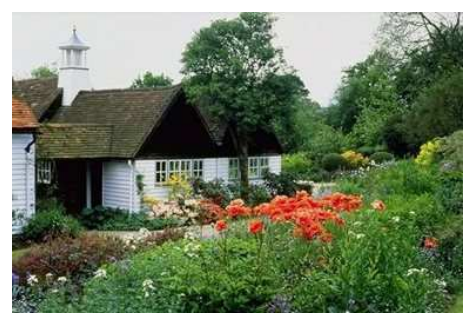

flowers, garden, house, tower

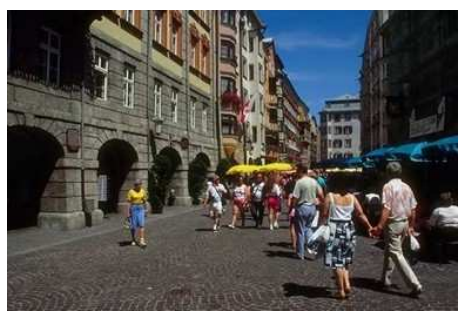

buildings, people, sky, street

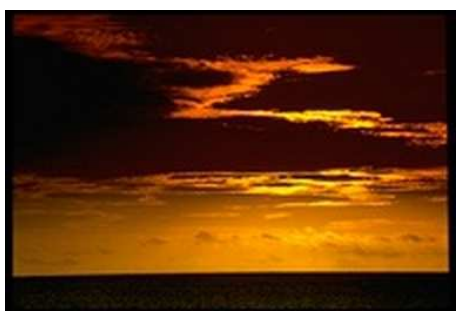

clouds, formation, sky, sunset

Fig. 1. Typical image captioning in the Corel Stock Photo Library.

images are ranked with respect to their similarity to this query index. Given that indices are directly derived from the image content, this process requires no semantic labeling. The QBE paradigm is therefore an interesting solution for particular domains such as medical imaging [10], satellite images, or personal photo collections [13], where the query effectively exists as an image. These data sources tend to be specific, as the corresponding QBE solutions.

QBE is not suitable for other types of image datasets. Commercial image collections such as Getty images or Corbis are searched with text-based queries because retrieval based on lowlevel visual similarity is, in general, not satisfactory for the user. The natural query process is in these cases textual, and images in a collection are therefore indexed with words. Despite the development of systems and tools to assist it, this textual indexing process involves a substantial amount of work and usually results in heavy costs. Automatic image annotation has thus emerged as one of the key research areas in multimedia information retrieval, as an alternative to costly, labor-intensive manual captioning [2], [3], [17], [12], [16], [18], [22], [9], [15], [26], [20].

Automatic image annotation systems take advantage of existing annotated image datasets to link the visual and textual modalities using machine learning techniques. While this framework seems very close to standard object detection [35], [1], key differences make automatic image annotation a distinct research problem. Although the vocabulary - the set of valid annotation words - might be constrained, captions from image collections can exhibit a large variability in general. Several words can describe one or more regions or even the whole image (see Figure 1), which differs from the standard object detection scenario. Furthermore, the development of classspecific features and classifiers [34] is difficult, as the vocabulary size is usually much larger than the number of classes in standard object detection problems. Automatic image annotation systems therefore tend to rely on generic features, and usually learn one model for the whole vocabulary [2], [3], [17], [12], [16], [22], [9], [15], [26], [20]. 
Independently of what features are chosen, the question is how to model the relation between captions and visual features to achieve the best textual indexing. A whole range of methods from a simple empirical distribution estimation to complex generative probabilistic models have been proposed in the literature, offering a large variety of approaches. However, the difference in the nature of text captions and image features has not yet been fully investigated and exploited. In general, the textual and visual modalities are either considered as equivalent sources of data [22], [9], [20], [17], [26], or the caption words are simply considered as a class label [31], [8], [18] instead of a modality as such. The CORR-LDA (Correspondence Latent Dirichlet Allocation) [3] model is a notable exception, that builds a language-based correspondence between text and images. It first generates a set of hidden variables (latent aspects) that generate the regions of an image, decomposing an image into a mixture of latent aspects. A subset of these latent aspects is then selected to generate the text caption, what intuitively corresponds to the natural process of image annotation.

The CORR-LDA model acknowledges the complementarity of text and images as sources of information, as well as their difference in carrying semantic content, which needs be taken into account to model the relation between modalities more accurately, with the goal of generating a better textual indexing. This paper investigates this concept, proposing a new dependence between words and image regions based on latent aspects. The contributions of our paper are the following. First, we present an alternative image representation to the standard Blob histogram, that combines quantized local color information and quantized local texture descriptors. Quantized versions of invariant local descriptors have been recently proposed as promising representations of objects and scenes [27], [11], [29], and applied to small number of classes. However, to our knowledge, this representation has not been previously used in the context of image annotation, a more challenging problem from the number of concepts that is addressed. The effect of each type of visual features and their combination is analyzed in details, and we prove their complementarity by demonstrating improvement of the retrieval performance for a majority of word queries for all the models that we consider. Second, we propose a probabilistic framework to analyze the contribution of the textual and the visual modalities separately. We assume that the two modalities share the same conditional probability distribution over a latent aspect variable, that can be estimated from both or one of the two modalities for a given image. In this way, equal importance can be given to the visual and the textual features in defining the latent space, or one 
of the two modalities can dominate. Based on extensive experiments, this framework allows us to show that the textual modality is more appropriate to learn a semantically meaningful latent space, what directly translates into an improved annotation performance. Finally, a comparison between different recently proposed methods is presented, and a detailed evaluation of the performance shows the validity of our framework.

The paper is organized as follows. Section II presents an overview of the research in automatic image annotation and contrasts it with our work. Section VI-A discusses the data and the visual representation considered in this work. Section IV describes our probabilistic framework for image annotation. In Section $\mathrm{V}$ we discuss state-of-the-art models that we implemented for comparison. Results and discussion are presented in Section VI.

\section{RELATED WORK}

Existing works in automatic image annotation can be differentiated by the way in which they represent images, and by the specific auto-annotation model. These two aspects are used to guide the discussion in the following paragraphs.

A common first step to all automatic image annotation methods is the image segmentation into regions, either using a fixed grid layout or an image segmentation algorithm. Regions have been described by standard set of features including spatial frequencies, color, shape and texture, and handled as continuous vectors [2], [3], [17], [12], [16], [18], or in quantized form [22], [9], [15], [26], [20]. Different statistical assumptions about these quantized or continuous representations and image captions have led to different models. A representative selection of recent approaches is presented here.

The original approach described in [22] is based on a fixed grid image segmentation and a vector quantization step. The color and texture representations of all training image blocks are quantized into a finite set of visual terms (visterms), which transforms an image into a set of visterms. All words attached to an image are attributed to its constituting visterms, and the empirical distribution of each word in the vocabulary given all visterms is computed from the set of training documents. A new image is indexed by first computing its building visterms and then averaging the corresponding posterior distributions over words.

Contrarily to [22], the work in [9] relies on the Normalized Cuts segmentation algorithm [28] to identify arbitrary image regions and build blobs. These blobs coarsely match objects or object 
parts, which naturally brings up a new assumption: the existence of an implicit one-to-one correspondence between blobs and words in the annotated image. The idea is borrowed from the machine translation literature, and considers the word and blob modalities as two languages. An Expectation-Maximization (EM) procedure to estimate the probability distributions linking words and blobs is proposed. Once the model parameters are learned, words can be attached to a new image region. This region naming process is comparable to object recognition, even if regions do not necessarily match objects, due to the obvious limitations of the segmentation algorithm. A new image is annotated by the most probable words given its constituting blobs.

The cross-media relevance model described in [15], also relies on a quantized blob image representation. However, unlike [9], it does not assume a one-to-one correspondence between blobs and words in images. Images are considered as sets of words and blobs, which are assumed independent given the image. The conditional probability of a word (resp. blob) given a training image is estimated by the count of this word (resp. blob) in this image smoothed by the average count of this word (resp. blob) in the training set. These posterior distributions allow the estimation of the probability of a potential caption (set of words) and an unseen image (set of blobs) as an expectation over all training images. This annotation system improves the performance w.r.t the machine translation method [9].

Linear algebra-based methods applied on the word-by-document and Blob-by-document matrices are proposed in [26] to estimate the probability of a keyword given a blob. The correlation and the cosine measure between words and blobs are investigated to derive these conditional probabilities. The use of a Singular Value Decomposition (SVD) of the word-by-document and blob-by-document matrices, weighted with the $t$-idf (term frequency - inverse document frequency) scheme, shows an improvement of the annotation performance over the original representation. A consistent improvement over the machine translation model [9] is shown.

In [17] and [12], the authors of [15] abandon the quantization of image regions. With the same conditional independence assumptions than in their previous model [15], the continuous image region representation, modeled by a Gaussian Mixture Model (GMM), improves the image annotation performance. An additional modification is proposed in [12], where a multipleBernoulli distribution for image captions replaces the multinomial distribution.

A statistical model of 600 image categories is proposed in [18]. Categories are labeled with multiple words, and images are manually classified in these categories. A two-dimensional 
Multi-resolution Hidden Markov Model (2D-MHMM) is learned on a fixed-grid segmentation of all category examples. The likelihood of a new image given each category's 2D-MHMM allows to select caption words for this image. This work is related to the model vector indexing approach [31], where one classifier (Support Vector Machine) is trained for each semantic concept (7 concepts), and used for the indexing of a new image. The Content-based soft annotation (CBSA) system [8] is also based on binary classifiers (Based Point Machines and SVMs) trained for each word (116 words are considered), and index a new image with the output of each classifier. The drawbacks are the learning of one classifier per word [31], [8], or of one model per set of words [18].

Different models to represent the joint distribution of words and image regions are discussed in [2], [3]. A hidden aspect variable is assumed in the data generative process, which links the textual and visual modalities through conditional relationships. This assumption translates into several variations of Latent Dirichlet Allocation (LDA) based mixture models. Images are represented as a set of continuous region-based image features, and modeled by Gaussian distributions conditioned on the aspects, while caption words are modeled with multinomial distributions. For instance, in the CORR-LDA model [3], words are conditioned on aspects that generated image regions. This additional constraint on word generation improves the overall annotation performance over less constrained LDA-based models.

A whole range of performance measures for automatic image annotation systems has been discussed in the literature. The quality of short image captions ( $\leq 5$ words), intended to be similar to human annotations, has been evaluated with different measures [8], [9], [26], [15], [2], [20]. Specifically, the retrieval of images based on these short captions is evaluated with the precision and recall values of the retrieved image sets for a number of given queries in [31], [26], [9], [15]. Alternatively, the ratio of the correctly predicted words per image divided by the number of words in the ground truth annotation has also been used for the evaluation of short captions [26], [2]. Proposed by [2], another measure for caption evaluation is the Normalized Score, which depends on the number of predicted words, and allows to estimate the optimal number of words to predict [20]. However, the main goal of image annotation is to allow textbased queries for image retrieval, and this does not require the creation of binary text captions. All approaches (binary classification, probabilistic model, linear-algebra-based) actually provide a confidence value for each word, that can be used for ranking all images in a collection. The 
confidence values for each word enables the construction of an image index, that can be used for text-based image retrieval [31], [8], [15]. The average precision of a query (see Section VI-B), summarized by the mean average precision (mAP) for a set of queries, is then the natural metric for the retrieval performance. This way of annotating/indexing images and evaluating retrieval performance has started to become consensual [31], [15], and we therefore use it in this paper.

As it should be clear from this overview, the existing approaches proposed to learn relationships between visual and textual modalities in annotated images differ in the way images are represented, in the dependence assumptions that are made between image regions and words, and in the way model learning is performed. In this paper, we propose a probabilistic framework related to [2] and [3] that includes a hidden aspect variable to link the visual and textual modalities. This approach allows to consider regions and words from an image jointly, contrarily to [22], where image regions are considered independently, and to [18] and [31], where categories (or words) are treated independently. Moreover, given that only one model is learned for all the words in the vocabulary, this type of approach might be better suited for large vocabularies than the supervised learning procedures proposed in [18], [31], which need to learn one model for each word. Finally, words and image features are of different nature and carry quite distinct level of semantics, and so we believe that these differences should influence how these two modalities are learned. Words and blobs are assumed equivalent in [9] (translation between two languages), and are treated equivalently in some of the models described in [2] and [3]. Unlike these works, we investigate different possibilities of learning the two modalities jointly while changing their respective influence.

In this sense, the closest work to ours is CORR-LDA, which first samples a latent aspect variable to generate an image region from a conditional Gaussian distribution, and then samples an aspect from the same set of aspects to select a word from a conditional multinomial distribution. In contrast, in our work we do it differently because we use multinomial distributions conditioned on aspects to model the discrete visual features, with the possibility to model a similar data generative process as CORR-LDA, or to first generate the words and learn the related aspect distributions that we later link to the visual features. As stated in the introduction, we also propose an enriched image representation that includes quantized local image descriptors that has not been investigated in auto-annotation, but used in very recent work for scene and object classification [11], [29], [27]. We conduct a thorough study comparing various competitive 


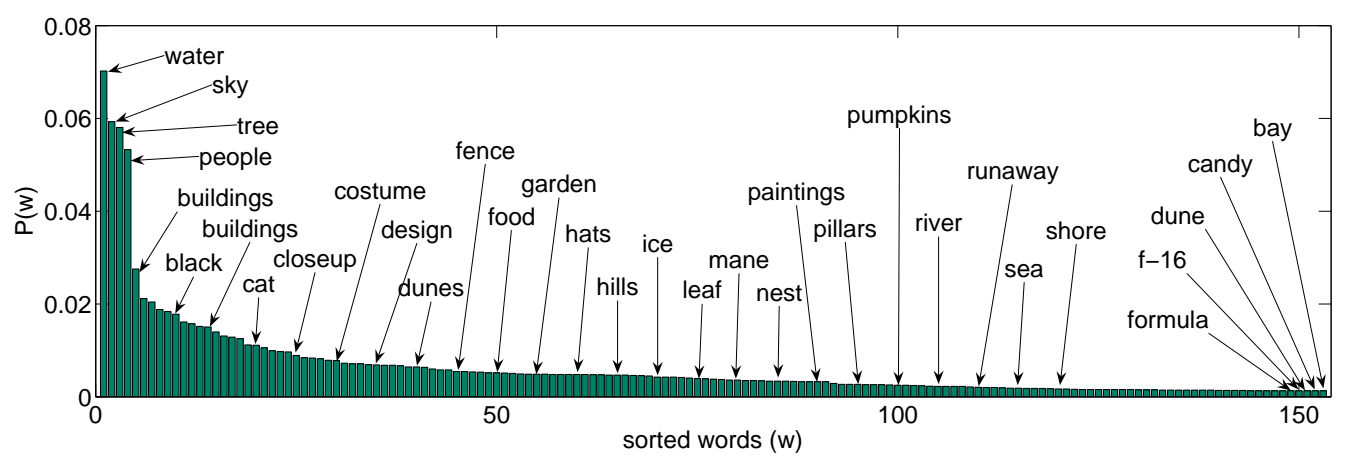

Fig. 2. Empirical distribution of words in the training images (set \#1). The most common words are water (1124), sky (949), tree (929), people (853), and buildings (441). The least common words are formula (21), f-16 (21), dunes (21), candy (21) and bay (21). The numbers in brackets indicate the number of images in which each word occurs. Other words are shown to illustrate the nature of the vocabulary.

methods with a consistent evaluation procedure, and show an improvement of performance.

\section{ANNOTATED IMAGE REPRESENTATION}

\section{A. Text caption representation}

Images in our dataset are annotated with a few unordered words selected from a vocabulary of size $N_{w}$. The representation of the caption of an image $d_{i}$ is an histogram $w\left(d_{i}\right)$ of size $N_{w}$ :

$$
w\left(d_{i}\right)=\left\{n\left(d_{i}, w_{1}\right), \ldots, n\left(d_{i}, w_{j}\right), \ldots, n\left(d_{i}, w_{N_{w}}\right)\right\},
$$

where $n\left(d_{i}, w_{j}\right)$ denotes the count of the word $w_{j}$ in the caption of the image $d_{i}$. This is a standard representation for text documents, that could also be used in the case of free-text captions after the word stopping and word stemming preprocessing steps. As shown in Figure 2, the distribution of words is highly skewed. As the dataset mainly consist of outdoor images, the words water, sky, tree, people, and buildings account for a big proportion of the probability mass. The empirical distribution also shows that there are many words represented by only a few training examples that nevertheless will have to be predicted, what advocates for a model that learns the co-occurrence of these infrequent words with more frequent words in order to predict them with higher accuracy. Training a separate model for a specific infrequent word seems difficult, while identifying the words with which this word co-occurs could be, instead, a good strategy. 


\section{B. Image representation}

We investigate three types of image representations based on quantized image regions, that are illustrated on Figure 3. The first relies on large-scale image regions, combining both texture and color information (see Figure 3(a)). The two other image representations are based on a larger number of smaller-scale image regions, uniformly extracted from a fixed grid (see Figure 3(b) or identified by a point detector (see Figure 3(c)). They capture color or texture information respectively. The three discrete feature types are described in the following.

1) Blobs: We consider an image representation originally proposed for region-based QBE [7], and later used for image annotation [2], [9], [3], [15]. A maximum of 10 regions per image, identified by the normalized cut segmentation algorithm [28], are represented by 36 features including color (18), texture (12), and shape/location (6). The K-means clustering algorithm is then applied to the region descriptors, quantizing them into a $N_{b}$-dimensional Blob representation. Note that the difference in the number of feature components makes the resulting Blob representation intrinsically biased towards color. An image $d_{i}$ is segmented into and a set of large image regions that are quantized and represented by the corresponding histogram $b\left(d_{i}\right)$ of size $N_{b}$ (see Figure 3(a)):

$$
b\left(d_{i}\right)=\left\{n\left(d_{i}, b_{1}\right), \ldots, n\left(d_{i}, b_{j}\right), \ldots, n\left(d_{i}, b_{N_{b}}\right)\right\}
$$

where $n\left(d_{i}, b_{j}\right)$ denotes the number of regions in image $d_{i}$ that are quantized into the Blob $b_{j}$. The motivation behind this representation is a possible match between the automatically segmented image regions and objects in the images. We see for instance on Figure 3 (a) that the green region matches trees in the original image, and that sky is covered by exactly one blob. As mentioned in [12], the match between the segmented regions and objects in the image is however relatively poor in general.

2) $H S:$ In spite of progress, no automatic segmentation algorithm is currently capable of dividing an image into consistently meaningful parts. The use of a segmentation algorithm is therefore difficult to justify, and we decided to extract image regions from a uniform grid, as illustrated in Figure 3 (b). The pixel color distribution from the resulting regions is represented by a 2D Hue-Saturation histogram, where the color brightness value from the Hue-SaturationValue (HSV) color-space is discarded for illumination invariance [25]. These HS histograms are then quantized into $N_{h}$ bins with the K-means clustering algorithm, to obtain the corresponding 

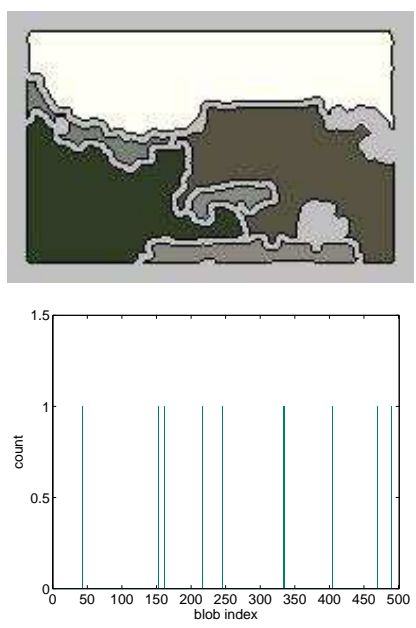

(a)
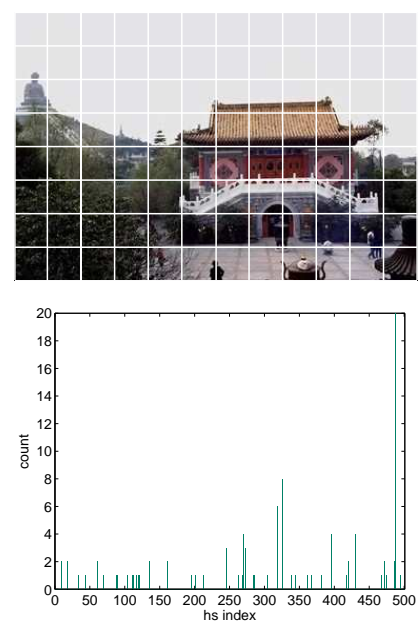

(b)

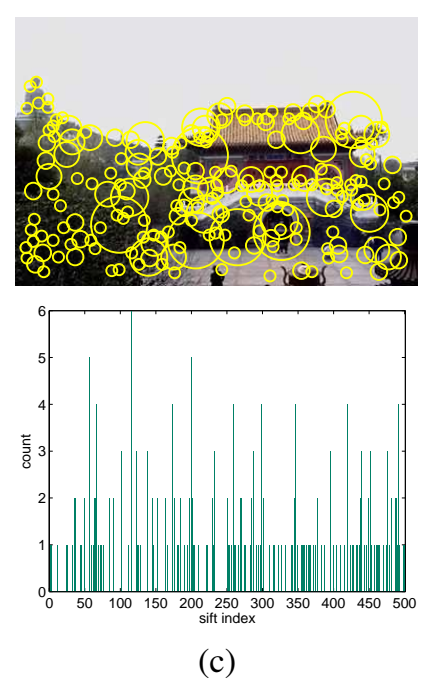

(c)

Fig. 3. Blob, HS, and SIFT image representations of the same image: (a) normalized cut image segmentation from which texture, color and shape features are extracted, and resulting histogram of the quantized image region features (Blobs), (b) uniform grid segmentation, color features are extracted, and resulting histogram of the quantized image region features (HS), (c) regions detected by the Difference-of-Gaussians (DoG) point detector, resulting histogram of the quantized SIFT descriptors.

histogram representation $h\left(d_{i}\right)$ of size $N_{h}$ for the image $d_{i}$ (see Figure 3(b)):

$$
h\left(d_{i}\right)=\left\{n\left(d_{i}, h_{1}\right), \ldots, n\left(d_{i}, h_{j}\right), \ldots, n\left(d_{i}, h_{N_{h}}\right)\right\}
$$

where $n\left(d_{i}, h_{j}\right)$ denotes the number of regions in image $d_{i}$ that are quantized into the HS bin $h_{j}$. Contrarily to a global color histogram, $h\left(d_{i}\right)$ encodes the distribution of color information from local image regions. In the rest of the paper, we refer to this representation of an image as the HS representation.

3) SIFT: We also propose the use of a third image representation based on local descriptors computed over automatically sampled image regions (see Figure 3(c)). Very recently, these features have been successfully combined with probabilistic latent space models [11], [29], [27] and have shown good performance in modeling different types of image content, including objects [29], [21] and scenes [11], [27], to, roughly speaking, a dozen concepts. However, its applicability to a much larger number of semantic concepts, to our knowledge, has not been investigated. In this representation, the image is first sampled with the difference-of-Gaussians (DoG) point detector [19], at different scales and locations (see Figure 3(c)). This detector has been built to be invariant to translation, scale, rotation, and illumination changes, and samples images at different locations and scales, depending on their content. We represent each detected regions with the Scale Invariant Feature Transform (SIFT) descriptor, which consists 
of a histogram of edge directions at different locations [19]. The SIFT descriptors are then quantized by the K-means clustering algorithm to obtain a discrete set of local $N_{s}$ image-patch indices. An image $d_{i}$ is represented by the histogram $s\left(d_{i}\right)$ of its constituting local patches (see Figure 3(c)):

$$
s\left(d_{i}\right)=\left\{n\left(d_{i}, s_{1}\right), \ldots, n\left(d_{i}, s_{j}\right), \ldots, n\left(d_{i}, s_{N_{s}}\right)\right\},
$$

where $n\left(d_{i}, s_{j}\right)$ i the number of local descriptors in the image $d_{i}$ that have been quantized into the image patch $s_{j}$. In the rest of the paper, we refer to this representation as the SIFT representation.

The Blob, HS, and SIFT image representations encode different image properties, and are therefore expected to produce different performance. The Blob representation is based on the joint quantization of shape, texture and color features, extracted from large image regions. The HS and SIFT representations are respectively based on the quantization of color or texture information, extracted from small-scale image regions. As we show on Figure 3, the number of regions that are considered in each case also varies: a maximum of 10 regions per image in the Blob case, 96 32-by-32-pixel square regions in the HS case, and an average of 240 detected points (depending on the image content) in the SIFT case. This makes the Blob histogram more sparse than the HS and SIFT histograms for an equivalent number of $500 \mathrm{~K}$-means clusters, as shown in Figure 3 (a,b, and c). In section VI, we investigate the combination of these image representations. For instance, using a direct concatenation of them in a first evaluation, the concatenation of the HS and SIFT features forms the complementary $v\left(d_{i}\right)=\left\{h\left(d_{i}\right), s\left(d_{i}\right)\right\}$ histogram of size $N_{v}=N_{h}+N_{s}$. To take advantage of these complementary source of visual information, the methods have to treat these unbalanced representations efficiently.

\section{LINKED ASPECT MODELS FOR ANNOTATED IMAGES}

\section{A. Aspect models for text}

An intuitive way to describe a text document is by considering the different topics it consists of. These topics are not explicit but can be derived from the documents themselves, and represent an accurate and compact summary of the original content. People usually compare text documents based on their respective topic distribution, and do not tend to evaluate similarity directly at the word level (unless looking for duplicates). To achieve a semantically meaningful indexing of texts, an increasing body of research in information retrieval aims at discovering methods to automatically identify hidden topics in a set of text documents. 
Different latent aspect models [14], [6], [4] have been proposed to achieve this task. Their common assumption is the sampling of a hidden variable (referred to as latent aspect) in the generative process of words in a document. Documents from the same text corpora share these latent aspects: a document is a mixture of latent aspects. These latent aspects are defined by multinomial distributions over words that are learned for each text corpora considered. These distributions characterize the aspects and show that a correspondence between topics identified by humans and latent aspects can exist [14], [4].

Model parameters are estimated from the co-occurrence of words across documents and learning is therefore fully unsupervised. The distribution over aspects represents an alternative text document representation, and can improve the classification of text documents into categories [4], or provide a less ambiguous representation for text retrieval applications [14].

\section{B. Aspect models for annotated images}

The concept of latent aspects is not restricted to text documents. Images are intuitively seen as mixtures of several content types, what makes them good candidates for a latent aspect approach. Different latent aspect models, adapted from the Latent Dirichlet Allocation [4] (LDA) model for text, have been proposed to model annotated images [2], [3]. A Gaussian distribution on the continuous visual feature space models the visual modality for each aspect, and different ways to combine the textual and the visual modalities using latent aspects are discussed. In particular, as presented in Section II, the Corr-LDA model [3] shares the latent aspects between the visual and textual modalities by first generating the image features, and then generate the words from the subset of aspects that generated the image features. In the Corr-LDA model, the visual modality thus drives the definition of the latent space to which the textual modality is then linked.

The Corr-LDA model shows that driving the definition of the latent aspects by the visual modality, and then sharing these aspects with the text modality is more appropriate than an unspecified, loose dependence between the two modalities. In our work, we investigate this key concept in more details, and compare the effect of a latent space driven by the visual features with a latent space driven by the textual features. We also compare the performance of these two options with the performance of a latent space learned from a concatenation of the two modalities. To conduct this analysis, three alternative procedures to learn a Probabilistic Latent Semantic Analysis model [14] (PLSA) for annotated images is proposed in Section IV-D. 
Although the LDA model has been shown to improve over PLSA in terms of perplexity [3] in text collections, we have chosen to base our investigation on PLSA since it allows for an exact EM algorithm. This makes the intended modifications of the learning procedure easier, without harming the resulting analysis. Moreover, PLSA has been recently shown to perform well on image classification tasks [27], [29], using the aspect mixture proportions to learn the classifiers. In particular, Sivic et al. [29] recently compared the PLSA and the LDA models for image classification, and they showed that a higher classification performance was obtained with PLSA in that case. We describe the PLSA model in Section IV-C, and our proposed three alternative methods to model annotated images with PLSA in Section IV-D.

\section{The PLSA model}

PLSA [14] assumes the existence of a latent aspect $z_{k}\left(k \in 1, \ldots, N_{z}\right)$ in the generative process of each element $x_{j}\left(j \in 1, \ldots, N_{x}\right)$ in a document $d_{i}\left(i \in 1, \ldots, N_{d}\right)$. Each occurrence $x_{j}$ is independent from the document it belongs to given the latent variable $z_{k}$, what corresponds to the joint probability expressed by:

$$
P\left(x_{j}, z_{k}, d_{i}\right)=P\left(d_{i}\right) P\left(z_{k} \mid d_{i}\right) P\left(x_{j} \mid z_{k}\right) .
$$

The joint probability of the observed variables is the marginalization over the $N_{z}$ latent aspects $z_{k}$ as expressed by:

$$
P\left(x_{j}, d_{i}\right)=P\left(d_{i}\right) \sum_{k}^{N_{z}} P\left(z_{k} \mid d_{i}\right) P\left(x_{j} \mid z_{k}\right) .
$$

The graphical model shown in Figure 4 illustrates the conditional independence assumptions of the PLSA model expressed in Eq. 5. A documents $d_{i}$ is first selected with the probability $P\left(d_{i}\right)$, which is proportional to the size of the document $d_{i}$, and an aspect $z_{k}$ is selected from the conditional probability distribution $P\left(z \mid d_{i}\right)$. Given the aspect $z_{k}$, an element $x_{j}$ is selected according to the conditional probability distribution $P\left(x \mid z_{k}\right)$. More details of the model are presented in the following paragraphs.

1) Model parameters: The conditional probability distributions $P\left(x \mid z_{k}\right)$ and $P\left(z \mid d_{i}\right)$ are multinomial given that both $z$ and $x$ are discrete random variables. The parameters of these distributions are estimated by the Expectation-Maximization algorithm [14]. For a document collection containing $N_{x}$ different elements, $P(x \mid z)$ is a $N_{x}$-by- $N_{z}$ table that stores the 


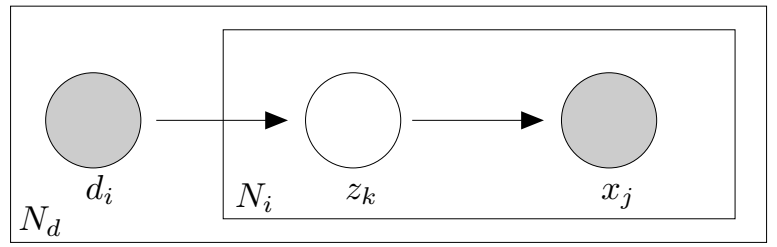

Fig. 4. The PLSA graphical model. Shaded nodes are observed. For each observation pair $\left(d_{i}, x_{j}\right), x_{j}$ is independent of $d_{i}$ given the latent aspect $z_{k} . N_{d}$ is the number of training documents $d_{i}$, and $N_{i}$ is the number of elements $x_{j}$ in $d_{i}$.

parameters of the $N_{z}$ multinomial distributions $P\left(x \mid z_{k}\right) . P\left(x \mid z_{k}\right)$ characterizes each aspect $z_{k}$, and is valid for documents that are not part of the training set. On the contrary, the $N_{z}$-by- $N_{d}$ table $P(z \mid d)$ is only relative to the $N_{d}$ training documents, as it stores the parameters of the $N_{d}$ multinomial distributions $P\left(z \mid d_{i}\right)$ that describes the training document $d_{i}$.

To illustrate these conditional probability distributions in the context of image captions, Figure 5(c) shows the PLSA decomposition of an image caption in $N_{z}=80$ aspects $\left(N_{z}\right.$ chosen arbitrarily), where the parameters are learned on the captions of 5188 images. The PLSA model decomposes the caption into three main aspects, which are represented in Figure 5 (d)-(f) by their multinomial distributions over words $P\left(w \mid z_{k}\right)$. As can be seen, aspect number 10 (Figure 5 (d)) is most likely to generate the word mountain (then valley); aspect number 3 (Figure 5 (e)) generates the words temple, statues and sculpture and stairs high probabilities; aspects 47 (Figure 5 (f)) is related to the words stone, ruins, sculpture, pillars and pyramids.

2) Learning: An Expectation-Maximization algorithm can be derived from the likelihood of the observed data (Eq.7) to estimate the parameters of the distributions $P(x \mid z)$ and $P(z \mid d)$.

$$
\mathcal{L}=\prod_{i}^{N_{d}} \prod_{j}^{N_{x}} P\left(d_{i}\right) \sum_{k}^{N_{z}} P\left(z_{k} \mid d_{i}\right) P\left(x_{j} \mid z_{k}\right)^{n\left(d_{i}, x_{j}\right)},
$$

where $n\left(d_{i}, x_{j}\right)$ is the count of element $x_{j}$ in document $d_{i}$. The two steps of the EM algorithm are the following:

E-step : the conditional probability distribution of the latent aspect $z_{k}$ given the observation pair $\left(d_{i}, x_{j}\right)$ is computed from the previous estimate of the model parameters.

$$
P\left(z_{k} \mid d_{i}, x_{j}\right)=\frac{P\left(x_{j} \mid z_{k}\right) P\left(z_{k} \mid d_{i}\right)}{\sum_{k}^{N_{z}} P\left(x_{j} \mid z_{k}\right) P\left(z_{k} \mid d_{i}\right)}
$$

M-step : The parameters of the multinomial distribution $P(x \mid z)$ and $P(z \mid d)$ are updated with 


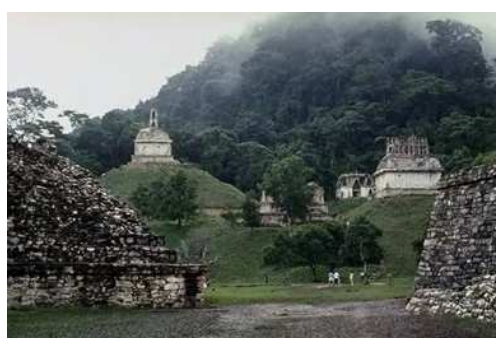

(a)

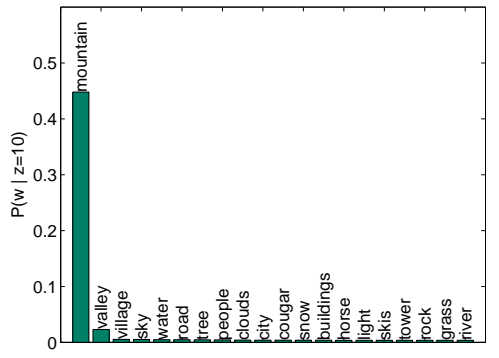

(d)

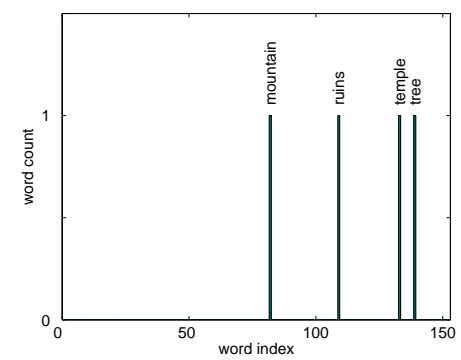

(b)

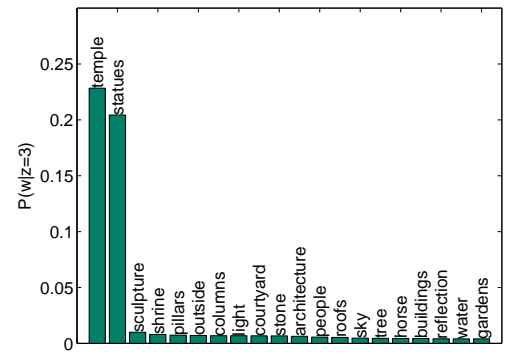

(e)

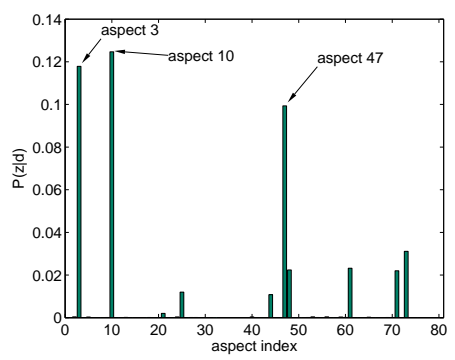

(c)

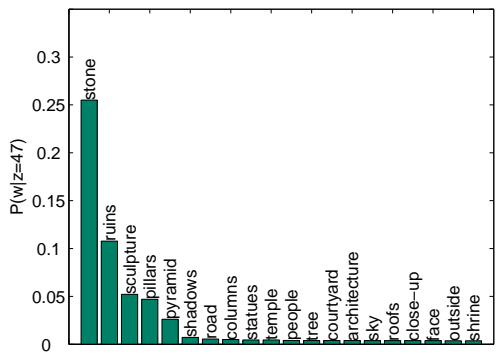

(f)

Fig. 5. Aspect decomposition of the image caption mountain, ruins, temple, and tree for a PLSA model trained with 80 aspects on 5188 image captions. (a) is the considered image $d_{i}$, (b) is the word caption histogram $w\left(d_{i}\right)$, (c) is the aspect distribution $P\left(z \mid d_{i}\right)$, and (d-f) are the distributions of the 20 top-ranked words given the three most probable aspects (10, 3 and 47 respectively).

the new expected values $P(z \mid d, x)$.

$$
\begin{aligned}
P\left(x_{j} \mid z_{k}\right) & =\frac{\sum_{i}^{N_{d}} n\left(d_{i}, x_{j}\right) P\left(z_{k} \mid d_{i}, x_{j}\right)}{\sum_{m}^{N_{x}} \sum_{i}^{N_{d}} n\left(d_{i}, x_{m}\right) P\left(z_{k} \mid d_{i}, x_{m}\right)} \\
P\left(z_{k} \mid d_{i}\right) & =\frac{\sum_{j}^{N_{x}} n\left(d_{i}, x_{j}\right) P\left(z_{k} \mid d_{i}, x_{j}\right)}{n\left(d_{i}\right)}
\end{aligned}
$$

3) Inference: PLSA of a new document: The conditional probability distribution over aspects $P\left(z \mid d_{\text {new }}\right)$ can be inferred for an unseen document $d_{\text {new }}$. The folding-in method proposed in [14], maximizes the likelihood of the document $d_{\text {new }}$ with a partial version of the EM algorithm described above, where $P(x \mid z)$ is obtained from training and kept fixed (i.e., not updated at each M-step). In doing so, $P\left(z \mid d_{\text {new }}\right)$ maximizes the likelihood of the document $d_{\text {new }}$ with respect to the previously learned $P(x \mid z)$ parameters.

4) Overfitting control: We control the overfitting of the model by early stopping, based on the likelihood of a validation set. We consider the folding-in likelihood, that allows good performance prediction and overfitting control without the need for a tempered version of the EM algorithm [5]. The probability of aspects given each validation document $P\left(z \mid d_{\text {valid }}\right)$ is first estimated using the folding-in method, as described in Section IV-C.3. The folding-in likelihood 
of the validation set given the current parameters is then defined as:

$$
\mathcal{L}_{\text {valid }}=\prod_{i}^{N_{d_{\text {valid }}}} \prod_{j}^{N_{x}} P\left(x_{j} \mid d_{i}\right)=\prod_{i}^{N_{d_{\text {valid }}}} \prod_{j}^{N_{x}} \sum_{k}^{N_{z}} P\left(x_{j} \mid z_{k}\right) P\left(z_{k} \mid d_{i}\right) .
$$

\section{Modeling annotated images with PLSA}

We discuss here three alternatives to learn a PLSA model for the co-occurrence of visual and textual features in annotated images. The first is a direct application of PLSA to the early integration of visual and textual modalities [20]. The two others are based on a variation of the PLSA EM algorithm that constrains the estimation of the conditional distributions of latent aspects given the training documents from one of the two modalities only. This allows to choose between the textual and the visual modality to estimate the mixture of aspects in a given document, what constrains the definition of the latent aspects on one or the other modality.

1) PLSA-mixed: The PLSA-MIXED [20] model learns a standard PLSA model on a concatenated representation of the textual and the visual features $x=(w, v)$. Using a training set of captioned images, $P(x \mid z)$ is learned for both textual and visual co-occurrences to capture simultaneous occurrence of visual features and words. Once $P(x \mid z)$ has been learned, it can be used to infer a distribution over words for a new image as follows. The new image $d_{\text {new }}$ is represented in the concatenated vector-space, where all word elements are zero (no annotation): $x_{n e w}=\left(0, v_{\text {new }}\right)$. The multinomial distribution over aspects given the new image $P\left(z \mid d_{\text {new }}\right)$ is then computed with the partial PLSA steps described in Section IV-C, and allows the computation of $P\left(x \mid d_{\text {new }}\right)$. The conditional probability distribution over words $P\left(w \mid d_{\text {new }}\right)$ is extracted from $P\left(x \mid d_{\text {new }}\right)$, and allows the annotation of the new image $d_{\text {new }}$.

2) Asymmetric PLSA learning: We propose to model a set of annotated images with a PLSA model for which the conditional distributions over aspects $P\left(z \mid d_{i}\right)$ is estimated from one of the two modality only. Contrarily to PLSA-MIXED, which learns $P\left(z \mid d_{i}\right)$ from both the visual and the textual modalities, this formulation allows to treat each modality differently, giving more importance to the text captions or the image features in the latent space definition. We refer to this alternative learning algorithm as an asymmetric PLSA learning. Intrinsically, PLSA-MIXED assumes that the two modalities have an equivalent importance in defining the latent space, given that the latent space is learned from their concatenated representation. The only potential 
imbalance could result from a marked difference between the number of words and the number of visual features in the images, and these values are not freely controlled in practice.

An asymmetric PLSA learning gives a better control of the respective influence of each modality in the latent space definition. This concretely allows to model an image as a mixture of latent aspects that is either defined by its text captions or by its visual features, resulting in different mixtures. The aspect distributions $P\left(z \mid d_{i}\right)$ are learned for all training documents from one modality only (visual or textual modality), and are kept fixed for the other modality (textual or visual modality respectively). We refer to PLSA-FEATURES when the aspect distributions are learned on the visual features, and to PLSA-WORDS when the aspect distributions are learned on the image captions. In the following, we describe how the parameters are learned in the asymmetric learning case, and how the distribution over words is estimated for a new document.

\section{Learning parameters}

The description of the learning process is valid for the PLSA-FEATURES and the PLSA-WORDS approaches, but differs on which modality the multinomial distribution over aspects are learned for the training documents. The first and second modalities are therefore referred to as $x^{1}$ and $x^{2}$ respectively, and correspond either to the visual or to the textual features in the following.

1) The first modality is used to estimate the $N_{z}$ conditional distributions $P\left(x^{1} \mid z_{k}\right)$ and the $N_{d}$ conditional distributions $P\left(z \mid d_{i}\right)$ on the training set.

2) We consider that the aspects have been observed for the set of training documents, and estimate the $N_{z}$ conditional probability distributions $P\left(x^{2} \mid z_{k}\right)$ for the second modality, keeping $P(z \mid d)$ from above fixed. Note that this technique is computationally similar to the standard PLSA procedure for inference in unseen documents described in Section IVC, where $P(x \mid z)$ is kept fixed and $P(z \mid d)$ is computed by likelihood maximization. However, what we are trying to do is, conceptually speaking, very different.

The parameters of the $P\left(x^{1} \mid z_{k}\right)$ and $P\left(x^{2} \mid z_{k}\right)$ distributions are defining the latent aspects $z_{k}$ based on the visual and textual modalities respectively for PLSA-FEATURES: conversely for PLSA-WORDS. Early stopping is performed for each of the two learning steps described above. In the second step, the probability of aspects given the validation documents $P\left(z \mid d_{\text {valid }}\right)$ estimated from the first step are not re-estimated by folding-in.

\section{Annotation by inference}


Given new visual features $v\left(d_{\text {new }}\right)$ and the previously estimated $P(v \mid z)$ parameters, the conditional probability distribution $P\left(z \mid d_{\text {new }}\right)$ is inferred for a new image $d_{\text {new }}$ using the standard PLSA procedure for a new document (Section IV-C). Furthermore, the conditional distribution of the words given this new image is given by:

$$
P\left(w \mid d_{\text {new }}\right)=\sum_{k}^{N_{z}} P\left(w \mid z_{k}\right) * P\left(z_{k} \mid d_{\text {new }}\right)
$$

\section{BASELINE METHODS}

Three baseline models for image annotation are considered for comparison with our models. The first baseline consists in a visual comparison between the image to annotate and the training images, propagating their annotations based on this similarity. The two other methods correspond to the state-of-the-art performance in image annotation when the discrete, quantized Blob representation $b(d)$ is used [9], [15], [26], [20].

\section{A. Annotation propagation}

Intuitively, training images that are similar to a new image $d_{\text {new }}$ should be taken into account to generate its annotation. Our simplest baseline therefore consists in computing the similarity between the image $d_{n e w}$ and the training images, sequentially attaching their respective annotation to $d_{\text {new }}$ based on these similarities. Concretely, we compute the cosine similarity between the image $d_{\text {new }}$ and the $N_{d}$ training images $d_{i}$ based on their respective visual representations $v\left(d_{\text {new }}\right)$ and $v\left(d_{i}\right)$ :

$$
\operatorname{sim}_{\text {cos }}\left(v\left(d_{\text {new }}\right), v\left(d_{i}\right)\right)=\frac{\sum_{j}^{N_{v}} n\left(d_{\text {new }}, v_{j}\right) n\left(d_{i}, v_{j}\right)}{\sqrt{\sum_{j}^{N_{v}} n\left(d_{\text {new }}, v_{j}\right)^{2}} \sqrt{\sum_{j}^{N_{v}} n\left(d_{i}, v_{j}\right)^{2}}}
$$

The training images are ranked with respect to this similarity measure, and the probability of a word $w_{i}$ given $d_{\text {new }}$ is estimated by the inverse of the best ranked image according to Eq. 13 that contains the word $w_{i}$ :

$$
P\left(w_{i} \mid d_{\text {new }}\right) \propto\left(\operatorname{rank}\left(d_{\text {best }}\right)\right)^{-1}
$$

where $d_{b e s t}$ is the most similar image to $d_{\text {new }}$ in the training set that contains the word $w_{i}$, and $\operatorname{rank}\left(d_{\text {best }}\right)$ is the rank order of this image given $d_{\text {new }}$. The word probabilities are then normalized so that $\sum_{N_{w}} P\left(w \mid d_{\text {new }}\right)=1$. 


\section{B. Cross-media relevance model}

In [15], the annotation of an unseen image $d_{\text {new }}$ is based on the joint probability of all its $m$ constituting visual elements $v_{l}$ and the word $w_{j}$. This joint probability is estimated by its expectation over the $N_{d}$ training images,

$$
P\left(w_{j}, v_{1}, \ldots, v_{m}\right)=\sum_{i}^{N_{d}} P\left(d_{i}\right) P\left(w_{j}, v_{1}, \ldots, v_{m} \mid d_{i}\right)
$$

The visual elements are considered independent given an image $d_{i}$, which gives:

$$
P\left(w_{j}, v_{1}, \ldots, v_{m}\right)=\sum_{i}^{N_{d}} P\left(d_{i}\right) P\left(w_{j} \mid d_{i}\right) \prod_{l}^{N_{v}} P\left(v_{l} \mid d_{i}\right)^{n\left(d_{i}, v_{l}\right)},
$$

where $n\left(d_{i}, v_{l}\right)$ is the count of the visual element $v_{l}$ in the image $d_{i}$. The probability of a word $w$ in a training image $d_{i}$ is the likelihood of this word in this image combined with the likelihood of this word in all the training images. A fusion parameter $\alpha$ controls the importance of the image and the training set likelihoods:

$$
P\left(w_{j} \mid d_{i}\right)=(1-\alpha) \frac{n\left(d_{i}, w_{j}\right)}{\sum_{l}^{N_{v}} n\left(d_{i}, v_{l}\right)+\sum_{j}^{N_{w}} n\left(d_{i}, w_{j}\right)}+\alpha \frac{n\left(w_{j}, d\right)}{N_{d}},
$$

where $n\left(d_{i}, w_{j}\right)$ denotes the count of the word $w_{j}$ in the image $d_{i}, n\left(d_{i}, v_{l}\right)$ is the count of the visual element $v_{l}$ in the image $d_{i}, n\left(w_{j}, d\right)$ is the number of images in which the word $w_{j}$ appears, and $N_{d}$ is the number of training images. Similarly, the probability of a visual element given an image $d_{i}$ is estimated by its likelihood in this image smoothed by its likelihood in the training set, controlled by a parameter $\beta$ :

$$
P\left(v_{l} \mid d_{i}\right)=(1-\beta) \frac{n\left(d_{i}, v_{l}\right)}{\sum_{l}^{N_{v}} n\left(d_{i}, v_{l}\right)+\sum_{j}^{N_{w}} n\left(d_{i}, w_{j}\right)}+\beta \frac{n\left(v_{l}, d\right)}{N_{d}},
$$

where $n\left(d_{i}, v_{l}\right)$ denotes the count of the visual element $v_{l}$ in the image $d_{i}, n\left(d_{i}, w_{j}\right)$ denotes the count of the word $w_{j}$ in the image $d_{i}, n\left(v_{l}, d\right)$ is the number of images in which the word $v_{l}$ appears, and $N_{d}$ is the number of training images. The parameters $\alpha$ and $\beta$ are estimated on a validation set to optimize the model performance.

\section{Cross-media translation table}

In [26], a translation table $T_{\text {cos }}$ between words and quantized visual features is proposed. The word-by-image matrix is weighted with the tf-idf scheme to obtain the weighted matrix $D_{w}$ :

$$
D_{w}=\left(n\left(d_{i}, w_{j}\right) * \log \left(\frac{N_{d}}{n\left(w_{j}, d\right)}\right)\right)_{N_{d} \times N_{w}},
$$


where $n\left(d_{i}, w_{j}\right)$ is the count of the word $w_{j}$ in the image $d_{i}, n\left(w_{j}, d\right)$ is the number of documents the word $w_{j}$ appears in, $N_{d}$ is the number of training images, and $N_{w}$ is the size of the vocabulary. Similarly, the feature-by-image matrix is weighted with the $t f-i d f$ scheme to obtain the weighted matrix $D_{v}$ :

$$
D_{v}=\left(n\left(d_{i}, v_{l}\right) * \log \left(\frac{N_{d}}{n\left(v_{l}, d\right)}\right)\right)_{N_{d} \times N_{v}},
$$

where $n\left(d_{i}, v_{l}\right)$ is the count of the word $v_{l}$ in the image $d_{i}, n\left(v_{l}, d\right)$ is the number of documents the visual element $v_{j}$ appears in, $N_{d}$ is the number of training images, and $N_{v}$ is the size of the visual feature space. A Singular Value Decomposition (SVD) is applied on the $D_{w}$ and $D_{v}$ matrices, keeping the first $r$ eigenvalues which preserve $90 \%$ of the variance to suppress the noise in the data. Let the j-th column of the matrix $D_{w}$ be $d_{w j}$, and the l-th column of the matrix $D_{v}$ be $d_{v l}$. The cross-media translation table $T$ is defined by:

$$
T_{\text {cos }}=\left(\operatorname{sim}_{\cos }\left(d_{w j}, d_{v l}\right)\right)_{N_{w} \times N_{v}},
$$

where the cosine similarity function $\operatorname{sim}_{\cos }()$ is defined in Eq. 13. Normalizing $T_{\cos }$ by column, the annotation of a new image $d_{\text {new }}$ represented by its histogram $v\left(d_{\text {new }}\right)$ is given by:

$$
P\left(w \mid d_{\text {new }}\right)=T_{\text {cos }} \times v\left(d_{\text {new }}\right) .
$$

\section{RESULTS}

\section{A. Data}

As shown in [23] for the case of Query by example (QBE), contradictory rankings can be obtained if the performance evaluation is conducted on different data subsets, even if these subsets are created from the same original image collection. To prevent this possible inaccuracy, it is crucial to compare different systems on identical data, with clearly defined training and testing sets. We conduct our experiments on an annotated image dataset that was originally used in [2], and consists in ten samples of roughly 16000 annotated images. Each sample is split into a training and a testing set, with an average number of 5240 training and 1750 testing images. The average vocabulary size is 161 . The Blob representation, as well as the description of the different samples were downloaded from http://kobus.ca/research/data/jmlr_2003/. 


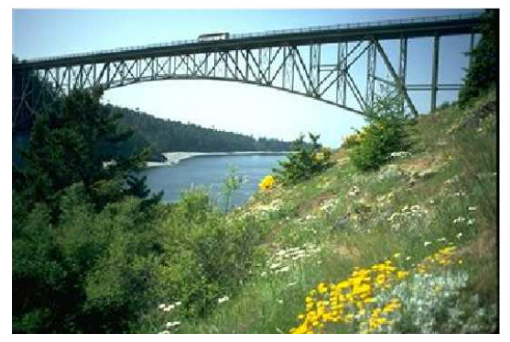

$\operatorname{arch}$

bridge

flowers
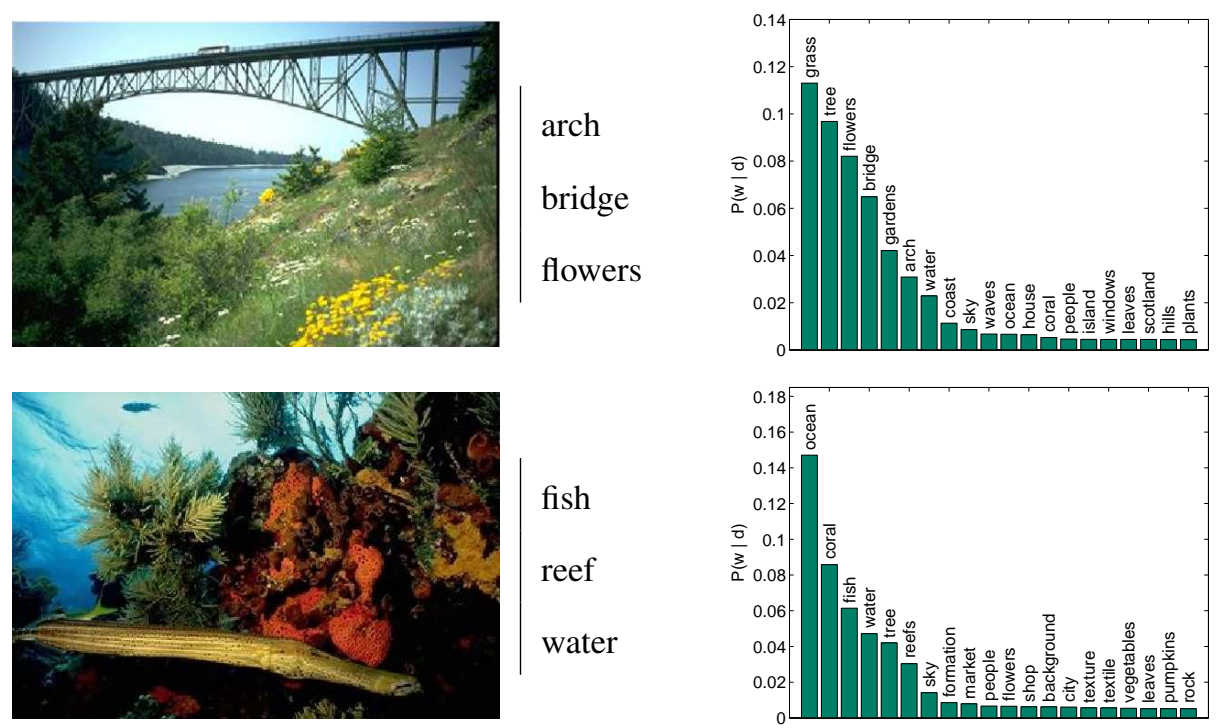

fish

reef

water

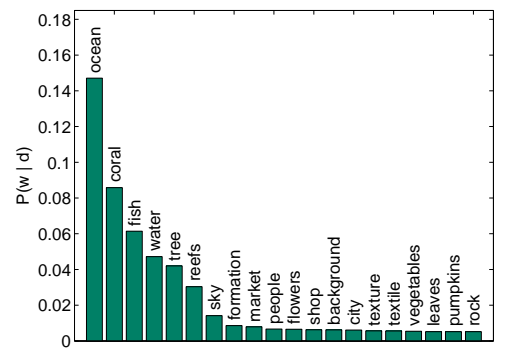

Fig. 6. The conditional probability distribution $P(w \mid d)$ inferred on two test images from their HS+SIFT representation with the PLSA-WORDS approach. The image and the ground truth annotation are shown on the left column, and the top twenty words and their conditional probability are shown on the right column.

\section{B. Mean average precision measure}

A number of works [9], [26], [12], [16], [15] measure the ability of the system to produce a human-like annotation, selecting a small number of words from the vocabulary. A fixed threshold or a fixed number of words has to be decided to extract short captions that can be used for image retrieval. With this, for a given query word, the number of correctly retrieved images divided by the number of retrieved images is the word precision, and the number of correctly retrieved images divided by the total number of correct images is the word recall. The average word precision and word recall values summarize the system performance.

One drawback of creating a human-like annotation is that only a fraction of words from the vocabulary are eventually predicted for the test images, because uncommon words tend not to be predicted due to a very low conditional probability. The word precision and recall values have thus to be presented together with the number of predictable words, as done in [9], [12], [16], [15], which makes the comparison between models unclear. Is it better for a system to predict only a few words with a high accuracy, or is a system more efficient if it can predict more words?

However, given that the goal is to index images for image retrieval, there is no need to produce such short, human-like annotation. The conditional probability distribution $P\left(w \mid d_{\text {new }}\right)$ can be 
used to rank the images for all possible queries. Even if the conditional probabilities of a specific word might be low for some of the images, the comparison of the relative probability values allows to rank the image collection for each word query. To illustrate this, the truncated word distribution inferred on two images using the PLSA-WORDS model are shown in Figure 6. The word flowers is in the top 20 words for both images, and the probability of the word flowers given the top image is higher than given the bottom image. This information would be discarded if the model is used to predict a fixed length annotation, although it can obviously be exploited for image ranking. The distribution over words in Figure 6 also shows how much more probable the word ocean is given the bottom image than given the top-image. This, however, would not be possible if we were only relying on a five-word annotation.

The performance measure used in this work is mean average precision (mAP). This is a standard measure for the retrieval of text documents for years, that has also been used by TRECVID to evaluate the semantic concept video retrieval task for several years (details can be found at http://www-nlpir.nist.gov/projects/trecvid/). mAP has the ability to summarize the performance in a meaningul way. To compute it, the average precision (AP) of a query $q$ is first defined as the sum of the precisions of the correctly retrieved words at rank $i$, divided by the total number of relevant images $\operatorname{rel}(q)$ for this query:

$$
m A P=\frac{\sum_{N_{q}} A P(q)}{N_{q}}, \text { where } A P(q)=\frac{\sum_{i \in \text { relevant }} \text { precision }(i)}{\operatorname{rel}(q)} .
$$

The average precision measure of a query is thus sensitive to the entire ranking of documents. The mean of the average precision of $N_{q}$ queries $q$ summarizes the performance of a retrieval system in one mean Average Precision (mAP) value:

\section{Hyperparameters and cross-validation}

We need to estimate two types of hyperparameters by cross-validation. The first one is the number of K-means clusters that defines the quantization of the visual features, the second one is the number of latent aspects for the approaches based on a PLSA model. The number of K-means clusters is cross-validated for the HS, SIFT and HS+SIFT representations, for 100, 200, 500, and 1000 clusters. The value of $N_{b}=500$ clusters for the Blob representation is kept fixed, as this representation is provided as is by the authors of [2]. The mAP performance of the Blob representation is given for comparison. The K-means models are learned on the 


\begin{tabular}{l||c||c|c|c|c||c|c|c|c}
\multicolumn{1}{c||}{} & \multicolumn{1}{c||}{ Blobs } & \multicolumn{9}{c||}{ HS } & \multicolumn{4}{c}{ SIFT } \\
\cline { 2 - 10 } & 500 & 100 & 200 & 500 & 1000 & 100 & 200 & 500 & 1000 \\
\hline propagation & $10.2(0.6)$ & $10.7(0.7)$ & $10.8(0.6)$ & $11.7(0.7)$ & $12.4(1.0)$ & $10.7(0.9)$ & $11.4(0.6)$ & $12.2(0.8)$ & $13.0(0.8)$ \\
CMRM [15] & $12.1(0.8)$ & $13.4(1.0)$ & $14.2(0.9)$ & $14.4(1.1)$ & $14.5(1.2)$ & $11.6(0.9)$ & $12.7(1.0)$ & $12.3(1.6)$ & $10.0(2.0)$ \\
SVD-cos [26] & $15.6(0.7)$ & $14.1(1.0)$ & $15.4(1.0)$ & $16.4(1.1)$ & $17.1(1.1)$ & $10.0(0.8)$ & $11.6(0.8)$ & $12.8(0.9)$ & $14.3(0.9)$
\end{tabular}

TABLE I

AVERAGE MAP VALUES (\%) OVER 10 CROSS-VALIDATION RUNS FOR DIFFERENT QUANTIZATION OF THE HS AND THE SIFT IMAGE REPRESENTATIONS, FOR THE THREE BASELINE METHODS. THE STANDARD DEVIATION IS GIVEN IN PARENTHESES.

\begin{tabular}{l||r|r|r|r}
\multicolumn{1}{c||}{} & \multicolumn{4}{c}{ HS+SIFT } \\
\cline { 2 - 5 } & $500-500$ & $500-1000$ & $1000-500$ & $1000-1000$ \\
\hline propagation & $16.0(1.3)$ & $15.5(1.4)$ & $16.8(1.2)$ & $16.4(1.3)$ \\
CMRM [15] & $17.6(0.8)$ & $17.4(0.8)$ & $6.2(0.8)$ & $4.8(0.8)$ \\
SVD-cos [26] & $19.9(1.5)$ & $20.9(1.7)$ & $20.2(1.7)$ & $21.2(1.7)$ \\
& \multicolumn{5}{c}{ TABLE II }
\end{tabular}

AVERAGE MAP VALUES (\%) OVER 10 CROSS-VALIDATION RUNS FOR REPRESENTATIONS BASED ON THE CONCATENATION OF DIFFERENT QUANTIZATION OF THE HS AND SIFT FEATURES, FOR THE THREE BASELINE METHODS. THE STANDARD DEVIATION IS GIVEN IN PARENTHESES.

training images of each sample set. On Table I, we show the mAP values obtained with the three baseline methods (propagation, CMRM, SVD-COS), averaged over ten cross-validation runs for one of the 10 sample sets. The hyperparameter values estimated by cross-validation from this sample set will be used for the remaining 9, as one set is assumed to be representative of the entire set. For the three baseline methods, the best number of K-means clusters for both HS and SIFT representations is 1000, except for the SIFT representation in the CMRM case, for which 200 clusters corresponds to the best retrieval performance. We also observe that the HS representation consistently achieves higher performance than the Blob representation for the same number of clusters. We use these estimated number of clusters for the final performance evaluation in Section VI-D.

We also estimated the number of clusters by cross-validation for the HS+SIFT concatenation, as reported on Table II. We restricted our analysis to the combination of HS and SIFT features for two reasons. First, as Table I suggests, the HS representation outperforms the Blob representation. Second, HS and SIFT features result from the quantization of local color-only and local textureonly information, respectively, while the Blob representation corresponds to the joint quantization 
HS
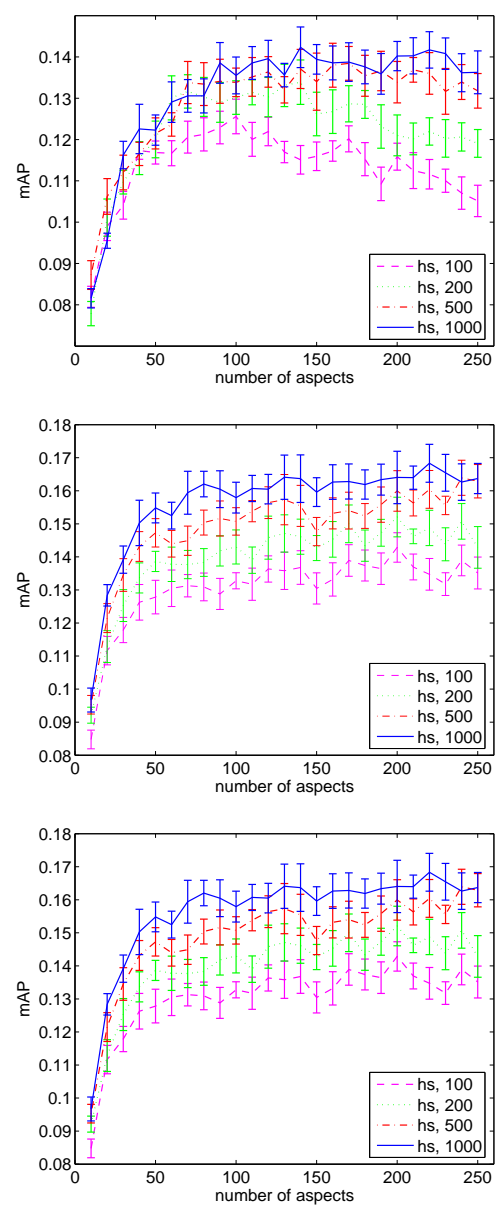

SIFT
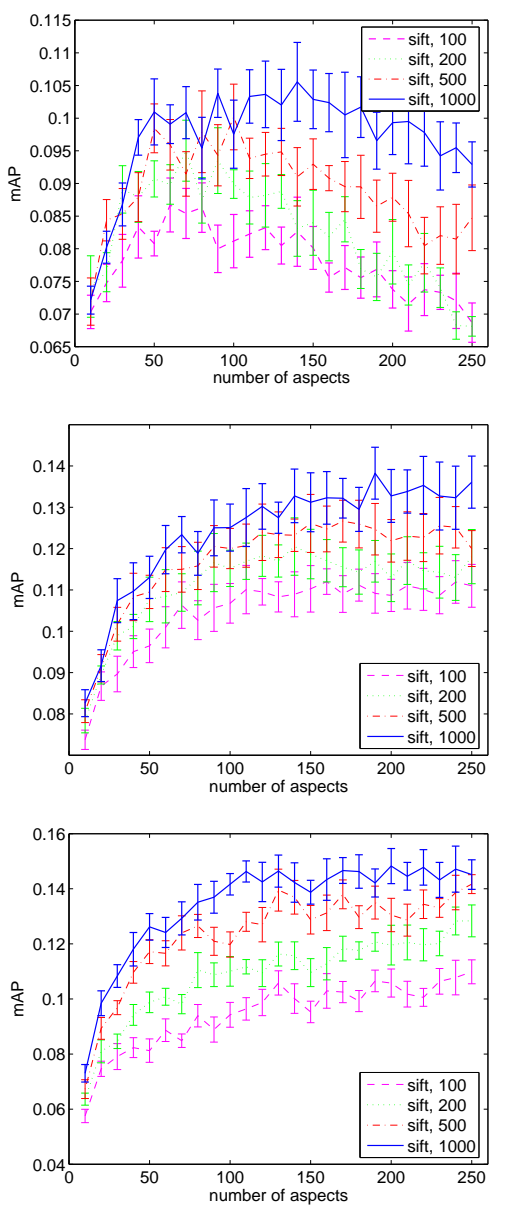

HS+SIFT
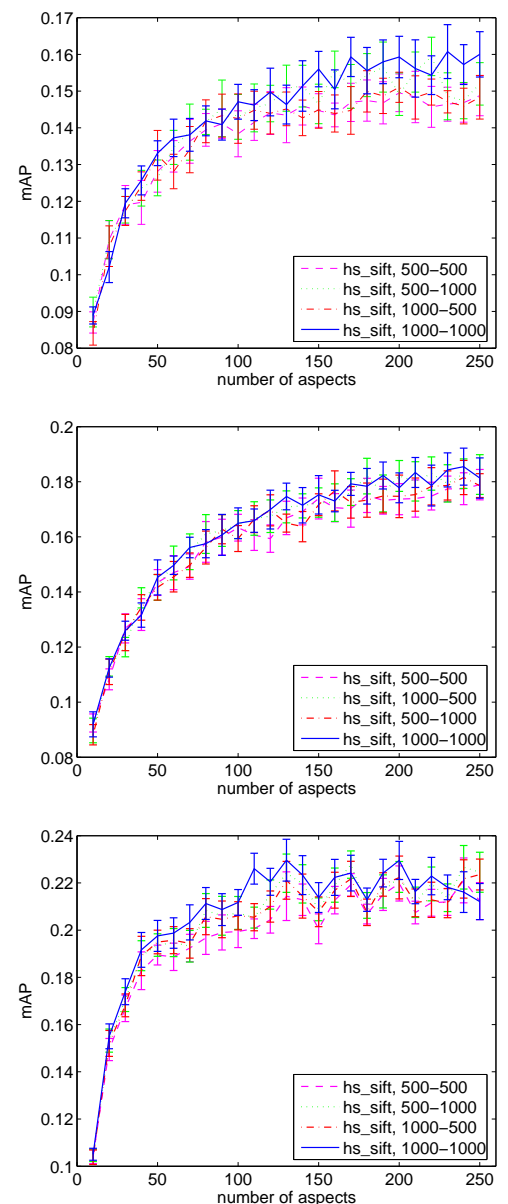

Fig. 7. Joint cross-validation of the number of aspects and the number of K-means clusters for the the HS (left column), SIFT (middle column), and HS+SIFT (right column) representations, and for the three PLSA learning methods. The mAP value obtained for PLSA-MIXED (top row), PLSA-FEATURES (middle row), and PLSA-WORDS (bottom row) are given. The error bars show the standard deviation of the mAP values for ten runs.

of color, texture and shape. Analyzing the effect of the combination of separately extracted color-only and texture-only information seems more intuitive than analyzing the combination of a texture-based representation with a joint color-texture-shape representation. The values from Table II show that the optimal $\left(N_{h}, N_{s}\right)$ HS-SIFT combination for the propagation method is $(1000,500),(500,500)$ for the CMRM case, and $(1000,1000)$ for the SVD-COS case. The results from the CMRM method drop significantly for the $(1000,500)$ and $(1000,1000)$ combination, although we carefully selected the $\alpha$ and $\beta$ parameters.

Regarding the PLSA-based approaches, as we have mentioned, they require the number of latent aspects $N_{z}$ to be estimated, as this hyperparmeter defines the capacity of the model: the 
number of parameters $\left.P=\left(N_{d}\left(N_{z}-1\right)\right)+\left(N_{z}\left(N_{x}-1\right)\right)\right) \sim N_{z}\left(N_{d}+N_{x}\right)$ linearly depends on $N_{z}$. The best value for the number of clusters therefore needs to be jointly estimated with the number of latent aspects for the three PLSA-based approaches, which is presented in Figure 7. The average of the mAP values computed for 10 cross-validation runs are reported on Figure 7 , where the number of latent aspects is varied between 10 to 250, for the three PLSA-based approaches. The number of K-means clusters for quantizing the visual features is also varied, and reported as a different line on each plot. The standard deviation over 10 cross-validation runs is shown with error bars.

The plots on Figure 7 allow to decide the number of aspects and the number of clusters given each PLSA learning methods and each image representation. The maximum number of K-means clusters seems to be a reasonable choice for the HS, SIFT and HS+SIFT representations. The results reported in Section VI-D are therefore computed with $N_{h}=1000$ and $N_{s}=1000$ clusters for the quantization of the HS and SIFT representations. Regarding the number of aspects $N_{z}$, the following values are chosen from the cross-validation experiments:

- PLSA-MIXED : $N_{z}=140$ for HS, $N_{z}=110$ for SIFT, and $N_{z}=170$ for HS+SIFT,

- PLSA-FEATURES: $N_{z}=170$ for HS, $N_{z}=150$ for SIFT, and $N_{z}=180$ for HS+SIFT,

- PLSA-WORDS : $N_{z}=120$ for HS, $N_{z}=110$ for SIFT, and $N_{z}=120$ for HS+SIFT.

\section{Overall performance}

The average of the mAP obtained on the 10 test sets with the hyperparameters estimated in Section VI-C are shown in Table III, where the performance of the Blob, HS, SIFT, and HS+SIFT representations for the six auto-annotation methods presented in Section IV-D and V are reported. The standard deviation of the mAP over the ten test sets is shown in parentheses. Note that the mAP values in Table III are consistently lower than the cross-validation values, because the retrieval tasks on which the mAP are now computed is more challenging: an average of 1750 images for test (vs. an average of 520 images for cross-validation) are ranked.

We see that the the PLSA-MIXED approach particularly fails to produce an efficient probabilistic indexing of the test images for all the image representations. In particular, its performance is lower than the simple propagation baseline that relies on a direct image similarity computation. Using a concatenated representation of words and visual features, PLSA-MIXED attempts to 


\begin{tabular}{l||r|r|r|r} 
& Blobs & HS & SIFT & HS+SIFT \\
\hline propagation & $7.8(0.7)$ & $9.0(0.2)$ & $9.4(1.0)$ & $13.1(0.5)$ \\
CMRM [15] & $11.5(1.1)$ & $10.7(1.1)$ & $7.9(0.5)$ & $13.4(1.0)$ \\
SVD-COS [26] & $12.9(1.1)$ & $12.9(0.8)$ & $10.7(0.7)$ & $16.6(1.1)$ \\
PLSA-MIXED & $5.8(0.8)$ & $10.2(0.8)$ & $7.5(0.6)$ & $11.9(1.3)$ \\
PLSA-FEATURES & $8.2(0.7)$ & $11.2(1.0)$ & $10.1(0.8)$ & $14.0(1.3)$ \\
PLSA-WORDS & $11.0(0.9)$ & $13.3(1.0)$ & $11.8(1.1)$ & $19.1(1.2)$
\end{tabular}

TABLE III

MAP VALUES (\%) FOR THE SIX METHODS WHEN COMBINATIONS OF HS AND SIFT FEATURES ARE USED.

simultaneously model the visual and textual modalities. It means that, intrinsically, PLSAMIXED assumes that the two modalities have an equivalent importance in defining the latent space, which as the results suggest, is not the most accurate assumption.

Except for the PLSA-MIXED case and the CMRM method when the SIFT representation is used, all methods achieve a higher performance than the propagation baseline. This shows that computing image similarity, although simple and intuitive, can only be considered as a low quality baseline for image annotation. It is, however, rather competitive w.r.t. the CMRM and PLSA-FEATURES methods, in particular for the HS and HS+SIFT image representations.

All methods take advantage of the HS+SIFT combination: the performance of a single feature type is always lower than their combination, which confirms that HS and SIFT features encode complementary information. It is interesting to notice that the CMRM and SVD-COS methods achieve the best performance for the Blob representation, which is the representation they were originally evaluated on [26], [15]. These methods however do not produce the best overall performance, especially when compared to the PLSA-WORDS method. Furthermore, when the conditional probability distributions of the aspects given the training documents $d_{i}, P\left(z \mid d_{i}\right)$, are learned from the visual features with PLSA-FEATURES, the estimation of the conditional distribution over words gives better results than PLSA-MIXED, but also lower mean average precision values than the baseline methods.

Regarding PLSA-WORDS, our method achieves a similar mAP performance than the SVDCOS method for the Blob representation, but it exploits the HS, SIFT, and the HS+SIFT representations more efficiently than both the CMRM and the SVD-COS approaches. Furthermore, it consistently performs better than CMRM. The PLSA-WORDS model achieves the best mAP overall score when the concatenated SIFT and HS+SIFT representations are used. In the HS+SIFT case, 


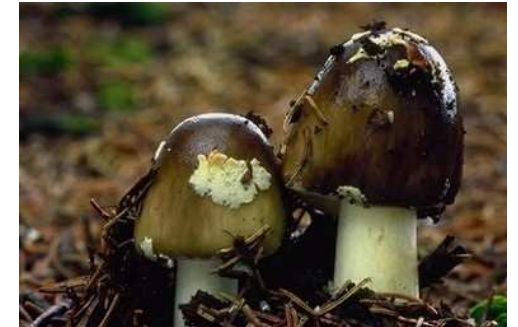

close-up, fungus, lichen, mushroom

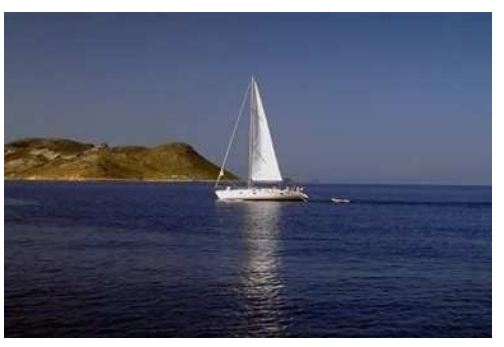

boats, coast, sails, water

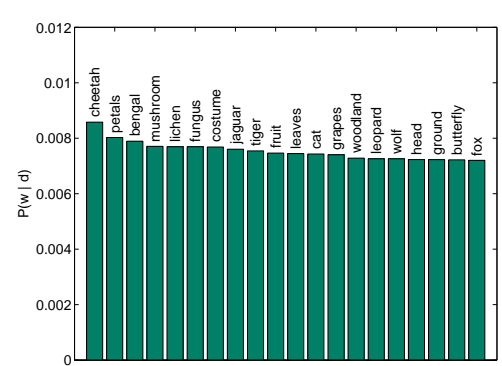

SVD-COS

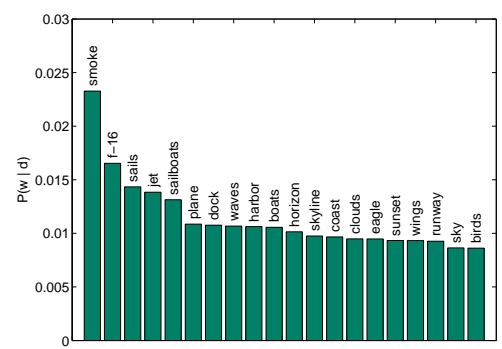

SVD-COS

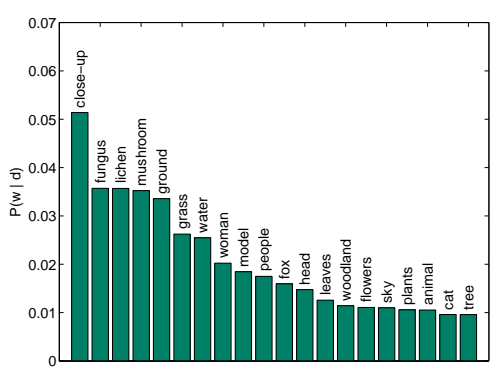

PLSA-WORDS

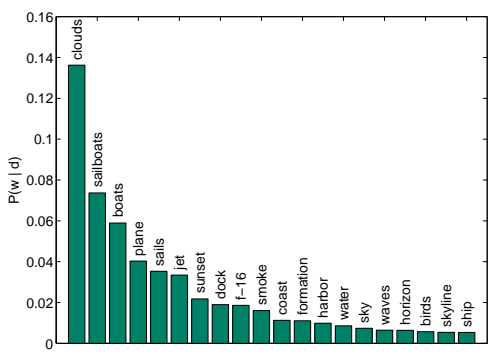

PLSA-WORDS

Fig. 8. The conditional probability distribution $P(w \mid d)$ inferred on two test images from their HS+SIFT representation with the SVD-COS and PLSA-WORDS approaches. The image and the ground truth annotation are shown on the left column, and the top twenty words and their conditional probability are shown on the middle and right columns for the SVD-COS and PLSA-WORDS approaches, respectively.

the PLSA-WORDS improves over the SVD-COS method by $15 \%$ (relative improvement). This improvement in performance is statistically significant according to a paired-samples T-test with a p-value of 0.05 , showing that the estimation of the aspect distribution based on the textual modality improves over both the linear algebra-based SVD-COS method and the method that does not use aspect variables. We illustrate the word distributions estimated by the SVD-COS and PLSA-WORDS approaches on Figure 8. For both images, the four words from the ground truth annotation are in the top 20 words given by PLSA-WORDS, while only three of them are in the top 20 words given by SVD-COS. More important, the probability values are more constrasted in the PLSA-WORDS case. A few words are sharing a large proportion of the probability mass, what will be an advantage for ranking images based on these values. The SVD-COS approach extimates flatter word distributions, making the probabilities of a given word very similar across images. More annotation examples are given at http://annotation.idiap.ch.

In the following two sections, we analyze the performance of PLSA-WORDS, the bestperforming model, in more details. 

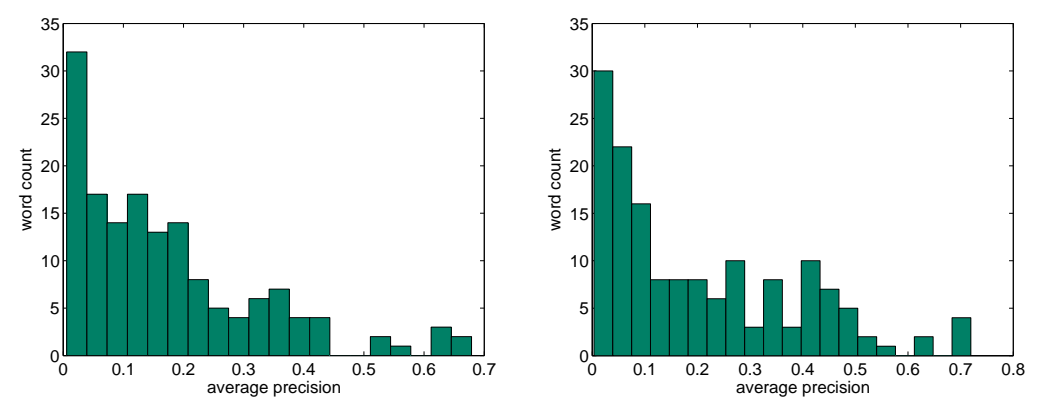

Fig. 9. Histogram of the 153 average precision values for SVD-COS (left) and PLSA-WORDS (right) methods.

\section{E. Per-word performance}

The histogram of the average precision values obtained with PLSA-WORDS in Figure 9 (right) shows a marked difference in performance for different words: half of the words have an average precision value higher than $0.14,65$ words have an average precision value below 0.1 , and 10 words have an average precision value above 0.5. A similar trend can be observed with the baseline methods, as shown for the SVD-COS method on Figure 9 (left). This important variation goes unnoticed if only the mean average precision is reported, as done in part of the existing literature [17], [12].

The combined effect of three factors could explain why the system does not rank images satisfactorily for some words while achieving a good performance for others. First, the number of training images per word ranges significantly in the dataset, from 21 (for bay, candy, formula, ...) to 1124 (water), and obviously the quality of a statistical model depends on the nature and the number of training examples. Second, all words have to be learned from the same set of visual features, which can be better suited for some concepts than for others. Third, the co-occurrence in text captions can have a combined influence with the two previous points; if a given word is correctly learned by the model because it is well represented by the visual features and has a sufficient number of training examples, other words that consistently co-occur with it could have a relatively high performance despite a low number of training examples. We investigate these three factors by analyzing individual word performance together with basic statistics from the training set.

The number of training images and the average precision for the 20 words with the best and the worst performance with the PLSA-WORDS model are shown in Figure 10, which shows that there is a difference in the average number of examples for the 20 best performing words 


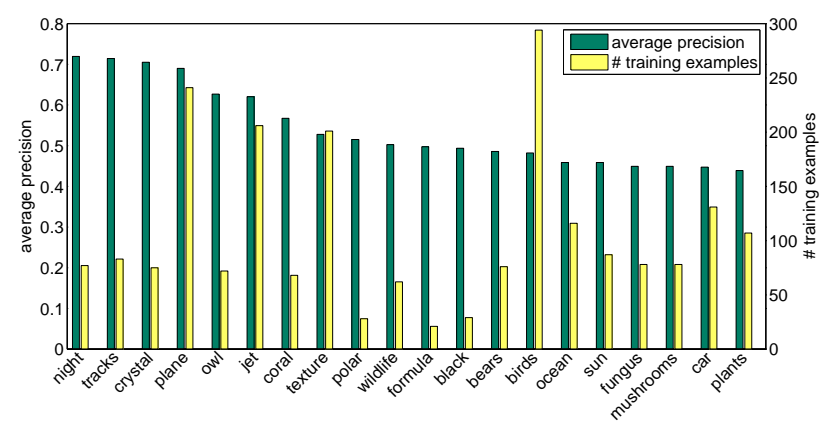

(a)

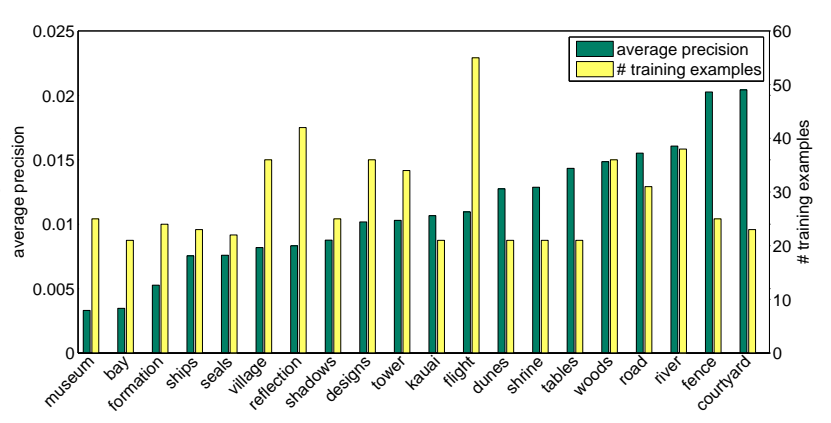

(b)

Fig. 10. Average precision and number of training examples for (a) the twenty best, and (b) the twenty worst average precision values (ranked in decreasing order in (a), and in increasing order in (b)) obtained with the PLSA-WORDS annotation.

compared to the 20 worst performing words. The former have 106 training examples on average, while the latter have an average of 29 examples. This fact suggests that the number of examples does indeed influence the performance of a word in general, because a low number of examples often does not allow to capture the statistical variations of a word appearance.

However, we also see in Figure 10 that, even though words have a comparable number of training examples, their respective performance is completely different. The words polar, formula, and black (Figure 10a) have a high average precision value $(\sim 0.5)$, while the words river, woods, and road (Figure 10b) are part of the 20 words with the lowest average precision $(\sim 0.015)$. The performance of a given word thus not only depends on the number of training examples, but also on the two other factors mentioned above.

In cases when the images a word is attached to depict consistent visual content that is well represented by the feature set, the model can learn the representation from little training data. For instance, images that are annotated with the word formula contain distinctive visual features that can be captured from a relatively small number of examples (21 in the dataset), while providing an high average precision value of 0.5 for this word. Similarly, the word polar is mainly attached to winter images which have a very distinctive white aspect, and is therefore well predicted (average precision of 0.51 ) despite very few training examples (only 28). On the contrary, the words reflection and museum for instance are not correctly modeled because the corresponding image content can not be learned properly from 25 and 42 examples, respectively.

For models such as PLSA-WORDS that learn co-occurrences in image captions, there is a possibility to improve the prediction of infrequent words from their co-occurring words. We show three examples of this effect on Figure 11, for the words skis, bridge and leaves. For these three 
(a)
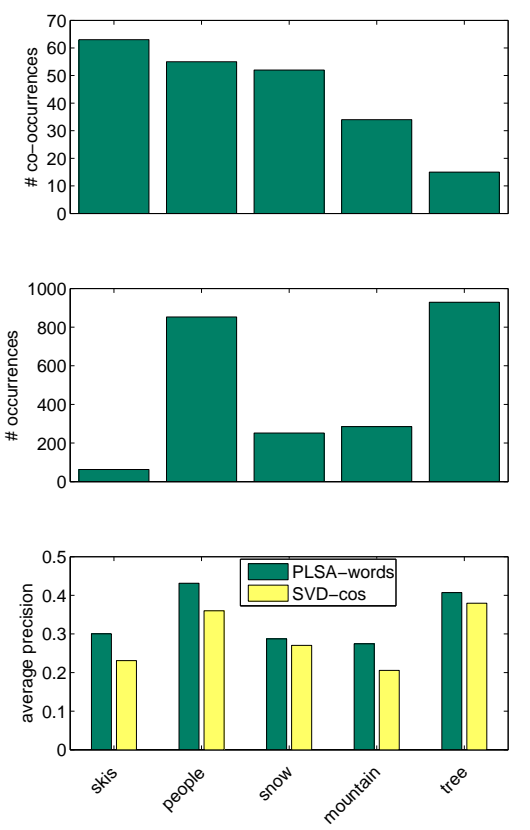

(b)
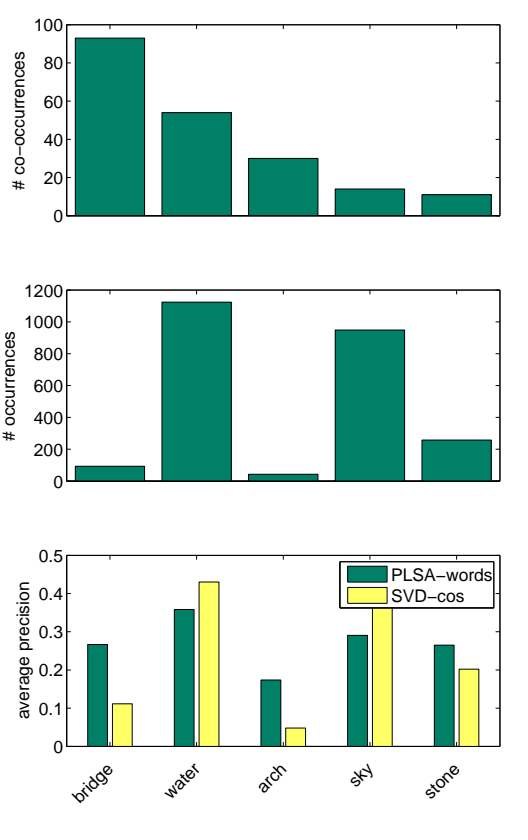

(c)
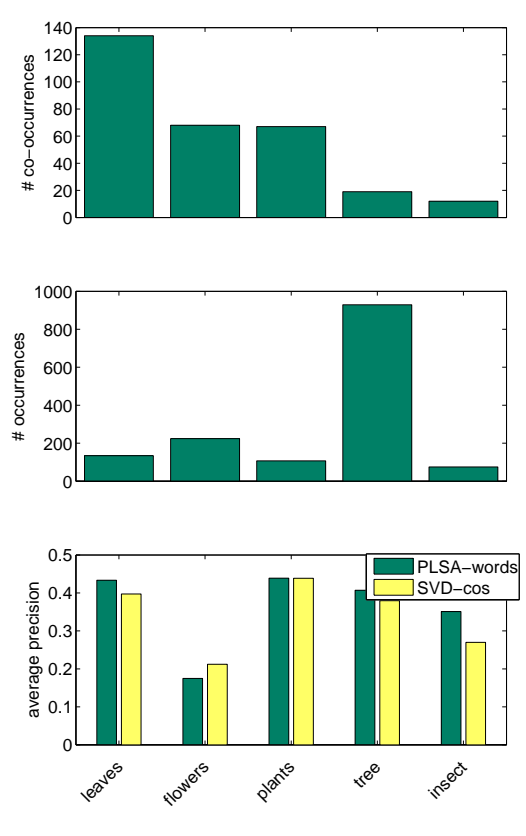

Fig. 11. Effect of word co-occurrences in captions for the words (a) skis, (b) bridge, and (c) leaves. The first row shows the number of times the four most frequently co-occurring words appear in the same caption as the word considered, the second row shows the total number of times each word appears in the training set, and the third row shows the average precision of these words for the PLSA-WORDS (green) and the SVD-cos (yellow) models.

words, the four words that co-occur the most with each of them are reported, as well as different statistics, including the number of times they co-occur with the word considered (top row), the number of times they appear in the training set (middle row), and their respective average precision (bottom row). Regarding the first example, although the word skis is only represented by 63 examples, the fact that it co-occurs quite often with more frequent words like people (which appears in 853 examples), snow (252 examples), and mountain (82 examples), allows PLSA-WORDS to predict skis with a high average precision (0.28). For the second example, the word bridge only has 93 examples, but is well predicted by the PLSA-WORDS model, because it co-occurs with words that have more examples in the training set, like water (which occurs in 1124 examples), sky (949 examples), and stone (258 examples). For the last example, the word leaves is predicted with an average precision of 0.43 by PLSA-WORDS, although there are only 134 leaves image examples. The fact that the word leaves co-occurs quite frequently with the words flowers (appearing in 224 examples), or tree (929 examples) also illustrates why a model that captures co-occurrence information at the caption level performs better than a model that 


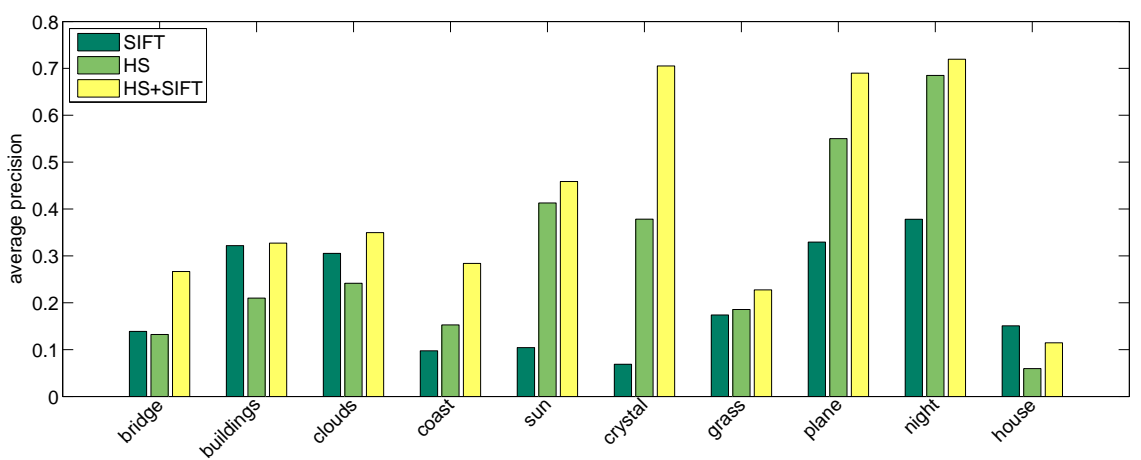

Fig. 12. Average precision of 10 selected words when SIFT (dark green), HS (green) and HS+SIFT (yellow) features represent the image. Depending on the word, the average precision values are higher for one of the two representations, and the combination of both improves in general.

does not model this information explicitly. In the two last examples, SVD-COS fails to take advantage of the co-occurrence with more frequent words, as PLSA-WORDS does.

\section{F. Combination of features}

To observe in more detail the benefit of combining HS and SIFT features for PLSA-WORDS, their individual and combined effects on the average precision of 10 representative words is shown in Figure 12. These 10 words are selected to illustrate different interesting behaviors that are observed when SIFT (dark green), HS (green) or both (yellow) are used.

As a general trend, we see that words that are rather well defined by color regions have higher average precision values when the HS representation is used, compared to SIFT local patches. In Figure 12, images annotated with words such as sun, crystal, plane, and night, depict colored regions, and are therefore well represented by the HS features. As shown in the Figure 12, the average precision of these words for a retrieval system based on the HS representation outperforms the same system based on the SIFT representation. This is a somewhat expected result. For instance, images annotated by the word sun present rather non-distinctive image structures, but contain very specific colors. Similarly, crystal images have a large variety of textures but present distinctive colors. The average precision of this word is therefore higher when HS features are used. The word plane also happens to be better represented by HS features as shown in Figure 12, which could be at first glance counter-intuitive. However, the word plane consistently appears in the context of blue sky, which is well identified by the HS representation.

On the contrary, if a word corresponds to images that contain specific textures, the SIFT representation becomes more informative and results in better image ranking. This can be 
observed in Figure 12, where the average precision values for the words buildings, clouds, and house, are higher when the SIFT (instead of the HS) representation is used. All these images contain structures that are poorly represented by HS elements, which encode color information. Based on local grayscale edge directions, the SIFT patches can efficiently depict parts of these structures, and allow to discriminate between e.g. white house and a polar bear that would be represented by a similar HS histogram. In Figure 12, we see that the house average precision values are more than two times bigger for the SIFT than for the Blob representation.

As already shown in Table III, the concatenation of the HS and SIFT representations provides the best ranking performance of the system. More precisely, it improves the average precision of 121 words compared to the SIFT-only representation, and 121 words compared to the HS-only representation. This complementarity can be analyzed in more details on the 10 words considered in Figure 12. The concatenation of HS and SIFT features improves the average precision of 9 of the 10 words in Figure 12 and on all of them on average, as shown in Table III.

Regarding limitations of the HS+SIFT combination, note that for some words, like house in Figure 12, combining the SIFT and the HS representations actually produces a worse image ranking than the SIFT-only case. This indicates that some ambiguity is introduced by the HS features in the related images, making them more similar to other images that are annotated with different words. Better mechanisms for data fusion could thus potentially improve the system performance, because a few words are better represented by one of the two feature types than by their simple concatenation. The fact that one model is learned for all the words does not allow a basic word-dependent weighting of the features, and more elaborate schemes have to be explored in the future.

\section{CONCLUSION}

We presented three alternative algorithms to learn a PLSA model for annotated images, and evaluated their ability for cross-media image indexing. The learning methods differ in which of the textual or the visual modality is dominant to learn the mixture of aspects for an image and its text caption, and these differences influence the accuracy of the inferred semantic indices. The best retrieval performance is achieved when the mixture of latent aspects is learned from the text captions (our PLSA-WORDS model), creating semantically meaningful aspects.

We also proposed to combine quantized local color information with quantized local image 
descriptors, demonstrating their complementarity and their improved performance when compared to the standard Blob representation. The performance of all the models was improved by the use of this combined image representation, that depicts an image as a set of local color-based regions and local texture-based regions. In particular, the PLSA-WORDS model achieved the best performance with respect to recent methods.

The quality of the image ranking greatly varies depending on the query, and we analyzed the possible factors in the case of the PLSA-WORDS model. Besides the difference in the number of training examples or the suitability of the visual features to represent a given concept, we have shown strong indications that PLSA-WORDS can take advantage of the co-occurrence of words in the text captions of the training images.

\section{ACKNOWLEDGMENTS}

This work was funded by the Swiss NCCR (IM)2. We thank Pedro Quelhas and Jean-Marc Odobez for discussions.

\section{REFERENCES}

[1] S. Agarwal, A. Awan, and D. Roth. Learning to detect objects in images via a sparse, part-based representation. IEEE Transactions on Pattern Analysis and Machine Intelligence, 26:1475-1490, 2004.

[2] K. Barnard, P. Duygulu, N. Freitas, D. Forsyth, D. Blei, and M.I. Jordan. Matching words and pictures. Journal of Machine Learning Research, 3:1107-1135, 2003.

[3] D. Blei and M. Jordan. Modeling annotated data. In Proc. of Int. Conf. on Research and Development in Information Retrieval (SIGIR), Toronto, Aug. 2003.

[4] D. Blei, A. Ng, and M. Jordan. Latent Dirichlet allocation. Journal of Machine Learning Research, 3:993-1022, 2003.

[5] Thorsten Brants. Test data likelihood for PLSA models. Information Retrieval, 8:181-196, 2005.

[6] W. Buntine. Variational extensions to EM and multinomial PCA. In Proc. of the European Conference on Machine Learning, London, Aug. 2002.

[7] C. Carson, S. Belongie, H. Greenspan, and J. Malik. Blobworld: Image segmentation using expectation-maximization and its application to image querying. IEEE Trans. on Pattern Analysis and Machine Intelligence, 24:1026-1038, 2002.

[8] E. Y. Chang, K. Goh, G. Sychay, and G. Wu. CBSA: Content-based soft annotation for multimodal image retrieval using bayes point machines. IEEE Transactions on Circuits and Systems for Video Technology, 13:26-38, 2003.

[9] P. Duygulu, K. Barnard, J.F.G de Freitas, and D.A. Forsyth. Object recognition as machine translation: Learning a lexicon for a fixed image vocabulary. In Proc. of Europ. Conf. on Computer Vision, Copenhagen, May 2002.

[10] J. Dy, C. Brodley, A. C. Kak, L. Broderick, and A. Aisen. Unsupervised feature selection applied to content-based retrieval of lung images. IEEE Transactions on Pattern Analysis and Machine Intelligence, 25:373-378, 2003.

[11] L. Fei-Fei and P. Perona. A Bayesian hierarchical model for learning natural scene categories. In Proc .of. IEEE Int. Conf. on Computer Vision And Pattern Recognition, San Diego, Jun. 2005. 
[12] S. L. Feng, R. Manmatha, and V. Lavrenko. Multiple Bernoulli relevance models for image and video annotation. In Proc. of IEEE Int. Conf. on Computer Vision and Pattern Recognition, Washington, Jun. 2004.

[13] A. Girgensohn, J. Adcock, and L. Wilcox. Leveraging face recognition technology to find and organize photos. In Proc. of the ACM SIGMM international workshop on Multimedia information retrieval, New York, 2004.

[14] T. Hofmann. Unsupervised learning by probabilistic latent semantic analysis. Machine Learning, 42:177-196, 2001.

[15] J. Jeon, V. Lavrenko, and R. Manmatha. Automatic image annotation and retrieval using cross-media relevance models. In Proc. 26th Int. Conf. on Research and Development in Information Retrieval (SIGIR), Toronto, Aug. 2003.

[16] J. Jeon and R. Manmatha. Using maximum entropy for automatic image annotation. In Proc. of IEEE Int. Conf. on Image and Video Retrieval, Dublin, Jul. 2004.

[17] V. Lavrenko, R. Manmatha, and J. Jeon. A model for learning the semantics of pictures. In Proc. of Advances in Neural Information Processing Systems, Vancouver and Whistler, Dec. 2003.

[18] J. Li and J. Z. Wang. Automatic linguistic indexing of pictures by a statistical modeling approach. IEEE Transaction on Pattern Analysis and Machine Intelligence, 25:1075-1088, 2003.

[19] D. G. Lowe. Distinctive image features from scale-invariant keypoints. International Journal of Computer Vision, 60:91110, 2004.

[20] F. Monay and D. Gatica-Perez. On image auto-annotation with latent space models. In Proc. ACM Int. Conf. on Multimedia, Berkeley, Nov. 2003.

[21] F. Monay, P. Quelhas, D. Gatica-Perez, and J.-M. Odobez. Constructing visual models with a latent space approach. In Proc. of the PASCAL Workshop on Subspace, Latent Structure and Feature Selection techniques: Statistical and Optimisation perspectives, Bohinj, Feb. 2005.

[22] Y. Mori, H. Takahashi, and R. Oka. Image-to-word transformation based on dividing and vector quantizing images with words. In Proc. of Int. Workshop on Multimedia Intelligent Storage and Retrieval Management, Orlando, Oct. 1999.

[23] H. Mueller, S. Marchand-Maillet, and T. Pun. The truth about Corel - evaluation in image retrieval. In Proc. of Int. Conf. on Image and Video Retrieval, London, Jul. 2002.

[24] W. Niblack, R. Barber, W. Equitz, M. Flicker, E. Glasman, D. Petkovic, P. Yanker, and C. Faloutsos. The QBIC project: query images by content using color, texture and shape. In Proc. of SPIE Conf. on Storage and Retrieval for Image and Video Databases, San Jose, Feb. 1993.

[25] M. Ortega, Y. Rui, K. Chakrabarti, S. Mehrotra, and T. S. Huang. Supporting similarity queries in MARS. In Proc. ACM Int. Conf. on Multimedia, Seattle, Nov. 1997.

[26] J.-Y. Pan, H.-J. Yang, P. Duygulu, and C. Faloutsos. Automatic image captioning. In Proc. of IEEE Int. Conf. on Multimedia and Expo, Taiwan, Jun. 2004.

[27] P. Quelhas, F. Monay, J.-M. Odobez, D. Gatica-Perez, T. Tuytelaars, and L. V. Gool. Modeling scenes with local descriptors and latent aspects. In Proc. of IEEE Int. Conf. on Computer Vision, Beijing, Oct. 2005.

[28] J. Shi and J. Malik. Normalized cuts and image segmentation. In Proc. of IEEE Int. Conf. on Computer Vision and Pattern Recognition, San Juan, Jun. 1997.

[29] J. Sivic, B. C. Russell, A. A. Efros, A. Zisserman, and W. T. Freeman. Discovering object categories in image collections. In Proc. of IEEE Int. Conf. on Computer Vision, Beijing, Oct. 2005.

[30] A. W. M. Smeulders, M. Worring, S. Santini, A. Gupta, and R. Jain. Content-based image retrieval: the end of the early years. IEEE transactions Pattern Analysis Machine Intelligence, 22:1349-1380, 2000. 
[31] J. Smith, C. Lin, M. Naphade, A. Natsev, and B. Tseng. Multimedia semantic indexing using model vectors. In Proc. of IEEE Int. Conference on Multimedia and Expo, Baltimore, Jul. 2003.

[32] J. R. Smith and S.-F. Chang. Visualseek: a fully automated content-based image query system. In Proc. of the ACM International Conference on Multimedia, Boston, Nov. 1996.

[33] K. Thieu and P. Viola. Boosting image retrieval. Int. Journal of Computer Vision, 56:17-36, 2004.

[34] P. Viola and M. J. Jones. Robust real-time face detection. Int. Journal of Computer Vision, 57:137-154, 2004.

[35] Paul Viola and Michael Jones. Rapid object detection using a boosted cascade of simple features. In Proc. of IEEE Int. Conf. on Computer Vision and Pattern Recognition, Hawaii, Dec. 2001. 